\title{
Longitudinal study of Mycobacterium avium ssp. paratuberculosis fecal shedding patterns and concurrent serological patterns in naturally infected dairy cattle
}

\author{
Nora Navarro-Gonzalez, ${ }^{1}$ Christine Fourichon, ${ }^{1}$ Philippe Blanquefort, ${ }^{2}$ Arnaud Delafosse, ${ }^{3}$ Alain Joly, ${ }^{4}$ \\ David Ngwa-Mbot, ${ }^{5}$ Franck Biet, ${ }^{6}$ Didier Boichard, ${ }^{7}$ Laurent Schibler, ${ }^{8}$ Laurent Journaux, ${ }^{9}$ Eric Meens, ${ }^{10}$ \\ and Raphael Guatteo ${ }^{1 *}$ \\ ${ }^{1}$ BIOEPAR, INRA, Oniris, 44307, Nantes, France \\ ${ }^{2}$ Groupement de Défense Sanitaire Pays de la Loire, 49800 Trelazé, France \\ ${ }^{3}$ Groupement de Défense Sanitaire Orne, 61000 Alençon, France \\ ${ }^{4}$ Groupement de Défense Sanitaire Bretagne, 56000 Vannes, France \\ ${ }^{5}$ Groupement de Défense Sanitaire France, 75578 Paris, France \\ ${ }^{6}$ ISP, INRA, Université de Tours, 37380, Nouzilly, France \\ ${ }^{7} \mathrm{GABI}$, INRA, AgroParisTech, Université Paris Saclay, 78350 Jouy-en-Josas, France \\ ${ }^{8}$ Allice, 75595 Paris, France \\ ${ }^{9}$ Idele, 75012 Paris, France \\ ${ }^{10}$ Groupement de Défense contre les Maladies des Animaux Seine Maritime, 76230 Bois-Guillaume, France
}

\section{ABSTRACT}

Mycobacterium avium ssp. paratuberculosis (MAP) is the etiological agent of paratuberculosis, a disease that affects ruminants worldwide. Despite global interest in the control of this disease, gaps exist in our knowledge of fecal shedding patterns and concurrent serological patterns. This longitudinal study in dairy cattle herds with high MAP seroprevalence in France aimed at accurately describing fecal shedding patterns over 1 year; relating those shedding patterns to individual animal characteristics (age, breed, parity); and exploring the association between fecal shedding patterns and serological patterns. To describe temporal fecal shedding patterns and continuity of shedding, along with the standard quantitative PCR (qPCR) threshold cycle we used a cutoff value that related to low or nonculturable fecal shedding. We also defined a threshold cycle indicative of shedding in high quantities to describe infection progression patterns. Twenty-one herds completed the study, and 782 cows were tested 4 times each. We obtained 4 sets of paired fecal qPCR and serum ELISA results from 757 cows. Although we targeted highly likely infectious animals, we found a large diversity of shedding patterns, as well as high variability between herds in the proportion of animals showing a given pattern. The fecal qPCR results of almost $20 \%$ of the final study sample were positioned at least once

Received October 24, 2018.

Accepted June 12, 2019.

*Corresponding author: raphael.guatteo@oniris-nantes.fr in the range that indicated low or nonculturable fecal shedding (between the adjusted and the standard cutoff value). Although these animals would typically be classified as non-shedders, they could be important to infection dynamics on the farm. Animals that shed at least twice consecutively and animals that shed in high quantities rarely reverted to negativity. Repeated fecal qPCR can be used to detect temporal fecal shedding traits, and the decision to cull an animal could practically be based on temporal, semiquantitative results. Overall, we found a mismatch between fecal shedding and ELISA seropositivity (637 animals were ELISAnegative 4 times, but only $13 \%$ of those animals were qPCR-negative 4 times). We found that having more than 2 ELISA-positive samples was strongly related to persistent and continuous shedding. We suggest that although serological testing is much less sensitive than qPCR, it can also be used, particularly over the course of multiple testing events, to identify animals that are most likely to contribute to the contamination of the farm environment.

Key words: Johne's disease, quantitative PCR, fecal culture

\section{INTRODUCTION}

Mycobacterium avium ssp. paratuberculosis (MAP) is the etiological agent of paratuberculosis, or Johne's disease, a granulomatous enteritis of ruminants. Cattle typically become infected during their first months via the fecal-oral route from contaminated farm environments. Infected animals go through a transitory phase of fecal shedding followed by a period of latency, and 
then a fecal shedding phase precedes the onset of clinical disease (Sweeney, 2011). However, large variability exists with respect to the start, duration, bacterial load, and persistence of fecal shedding. Clinical signs, including chronic diarrhea and emaciation, appear after a variably long incubation period (1 to $10 \mathrm{yr}$; Collins, 2003).

Paratuberculosis is of global interest because it negatively affects animal health and welfare. Infection with MAP causes important economic losses to the livestock industry and it poses concern as a zoonosis mainly for its potential role in human Crohn's disease, although this potential link is controversial (Waddell et al., 2016). Many countries have implemented paratuberculosis control programs, and some of these have been in place for decades (Geraghty et al., 2014).

Accurately detecting MAP infection is essential for control programs that incorporate a test-and-cull strategy based on the removal of the infected animals detected either by serology or by fecal shedding. A test-and-cull strategy in which all infected animals are culled may be not economically feasible on farms with a moderate to high prevalence, especially in endemic countries where reintroduction in the herd through the purchase of an infected animal is likely. Therefore, farmers need to establish priorities (for example, to cull the high shedders first and keep cows that are first-time positives or shed at a low level, or both). To aid in decision-making, predictors of the shedding trajectory that can be assessed on farm are needed, but to find these, we first need to understand all putative fecal shedding patterns derived from natural infections. So far, a diversity of MAP fecal shedding patterns has been reported, exacerbated by the different criteria used to define the putative shedding patterns. For example, Whitlock et al. (2000) classified cattle as high shedders, mid-shedders or low shedders as a function of their colony count at the first positive fecal sample. However, Nielsen (2008) established MAP fecal shedding groups (transient, low, high, and intermittent shedding) based on their colony count on an ordinal scale and on the persistence of shedding after the first positive sample detected. In reality, our knowledge of the diversity of fecal shedding patterns and their corresponding serological patterns is limited by difficulty in generating longitudinal data and imperfect diagnostic tools. Longitudinal MAP studies are resource- and labor-intensive, demand a large amount of funding, and require farmers' commitment. The limitations of the diagnostic tools (such as fecal culture or serology) are mostly associated with their low sensitivity in the early stages of infection (Nielsen and Toft, 2008).

Despite the existence of long-term, good-quality data that have advanced our knowledge (some examples are
Whitlock et al., 2000; Nielsen, 2008; Mitchell et al., 2015a), important gaps still exist in the description of MAP fecal shedding patterns. A limitation of past longitudinal studies is the relative low sampling frequency, which may miss rapid fluctuations that occur in the intervals (typically 6 mo to $1 \mathrm{yr}$ ) between sampling events in control plans. Khol et al. (2012) performed a monthly follow-up of MAP fecal shedding and antibodies for almost 1 yr to assess the outcome of commercial serology tests. However, the herd under study had a low prevalence ( $9 \%$ by real-time PCR), and more than $80 \%$ of the tested cows were MAP-negative in feces. Thus, no comprehensive description of fecal shedding patterns can result from such a study. The authors of another monthly follow-up with a 1-yr duration (Laurin et al., 2015) targeted known MAP-infectious cows to compare detection methods, but this study lacked serological testing and was based on 51 cows only. The most complete description of fecal shedding and serological patterns was done by Nielsen (2008), in which quarterly sampling in more than 1,900 cows from 8 dairy herds was conducted over 3 years. However, the study used fecal culture to detect MAP and may have underestimated the actual proportion of shedders, especially low shedders. Thus, data are still lacking to describe with precision the variability of MAP fecal shedding with respect to short-term variability (repeated samplings in a short time frame relative to a cow's life span) and quantitative accuracy [use of quantitative PCR (qPCR) for best sensitivity]. This longitudinal study in MAP-endemic dairy cattle aimed to (1) describe the MAP fecal shedding patterns detected by qPCR and the concurrent serological patterns; (2) explore whether there was an effect of age, breed, or parity on fecal shedding and serological patterns; and (3) test the association between serological patterns and fecal shedding traits.

\section{MATERIALS AND METHODS}

\section{Study Population and Study Sample}

Herd Selection Criteria. Twenty-two dairy herds located in the French region of Normandy were enrolled in this study according to 2 criteria: (1) showing a $\geq 8 \%$ MAP herd seroprevalence in the previous year (this information was available through the voluntary paratuberculosis control program); and (2) having at least 40 cows older than 24 mo (considered representative of the typical size of French dairy herds). With these criteria, we aimed at increasing the likelihood of detecting fecal shedders in the final study sample. None of the herds was vaccinated against paratuberculosis. Table 1 contains the detailed herd size, breeds, and age 
mean and range for each herd enrolled in the study. The first phase of the study (time 1) was conceived as a herd status assessment, followed by a selection of animals for quarterly follow-up. The sampling events took place between January 2011 and June 2012. The total number of cows older than 12 mo present in the enrolled herds at the beginning of the study was 2,838 . Feces and sera were collected from 2,706 cows. Access to nonlactating adults for the collection of samples was not possible because dry animals were kept on pasture. Results for both tests were available for 2,645 animals after discarding laboratory errors and animals missing any samples.

Animal Selection Criteria. Based on their serological and fecal shedding status at time 1, we selected a set of animals to continue in the follow-up (detailed criteria are shown in Table 2). Briefly, $100 \%$ of the animals that had an ELISA-corrected optical density $\left(\mathbf{O D}_{\mathbf{c}}\right)$ value, qPCR threshold cycle $\left(\mathbf{C}_{\mathbf{t}}\right)$ value, or both that highly suggested MAP infection were selected to favor inclusion of animals that were more likely to be shedders in the final study sample. We selected $50 \%$ of the animals with $\mathrm{OD}_{\mathrm{c}}$ or $\mathrm{qPCR}$ values that less confidently suggested MAP infection (that is, $50 \%$ of animals with $\mathrm{OD}_{\mathrm{c}}<10$ and $38 \geq \mathrm{C}_{\mathrm{t}}>35$, and $50 \%$ of animals with $30 \geq \mathrm{OD}_{\mathrm{c}} \geq 10$ and $\mathrm{C}_{\mathrm{t}}>38$ ). We selected only $20 \%$ of the animals with results that suggested MAP negativity at time 1 . In total, 1,475 animals met these criteria and were included in the set of animals to continue with follow-up. After the initial herd assessment, individual blood and fecal samples were collected from the selected individuals 3 more times, each time targeted in a 4-mo interval (range 3 to $9 \mathrm{mo}$ ).

Final Study Sample and Follow-Up. Of the 1,475 animals selected to continue with follow-up, 1,312 were sampled more than once, and 782 completed the 4 sampling events. Table 3 shows the number of animals per herd that successfully provided 4 data points for the description of fecal shedding patterns and serological patterns. Major causes that prevented us from continued sampling were the abandonment of the study at time 3 by herd $\mathrm{A}$, death or sale of animals, and cows being dried off and therefore sent to pasture, so that they could not be handled to collect samples. Specifically, 404 cows selected to continue the survey were culled or died during the study period. In the final study sample, the age at first sampling ranged from 12 to $137 \mathrm{mo}$; older ages were detected less frequently. In addition, because the interval from start to end date was not exactly 1 year for some herds, we checked the distribution of age at time 4 of the study. Only 3 animals in the final study sample were younger than 24 mo by the last sampling date, and they were retained for further analysis.
With respect to the sampling intervals, herds varied substantially in the completion of the schedule (Table 1 ), but most of the sampling events (53/61) occurred within $4 \pm 1$ mo. Table 3 shows the specific number of animals successfully assessed for MAP fecal shedding and blood antibodies in each herd and at each sampling event.

\section{Laboratory Methods}

Detection of MAP in Feces by $q P C R$. We used direct fecal qPCR to follow up on MAP shedding throughout the study. We extracted DNA from $1 \mathrm{~g}$ of feces using the NucleoSpin Tissue kit (Macherey-Nagel GmbH, Düren, Germany) following the manufacturer's instructions (https://www.mn-net.com/Portals/8/ attachments/Redakteure_Bio/Protocols/Genomic\%20 DNA/UM_gDNATissue.pdf). For this procedure, we used a positive control (MAP strain K-10) and a negative control (Mycobacterium smegmatis $\mathrm{mc}^{2} 155$ ). We identified MAP DNA using the Adiavet ParaTB Real Time kit (Adiagene, Saint Brieuc, France). We carried out qPCR according to the manufacturer's instructions (https://www.biox.com/en/download_en/373/NE045 -08.pdf/insert/0/). The qPCR assay used 2 primers and a Taq Man probe that were specific for MAP genetic sequence IS900. An internal control of synthetic DNA was included in each reaction to confirm the functionality of the qPCR assay.

In each case, the $C_{t}$ was recorded. We initially used the $\mathrm{C}_{\mathrm{t}}$ cutoff for MAP detection recommended by the manufacturer $\left(\mathrm{C}_{t}<40\right)$; this cutoff is hereafter referred to as the "standard cutoff."

Detection of MAP in Feces by Fecal Culture. A subset of 1,223 fecal samples collected at time 1 of the study was processed for fecal culture (FC), from which 1,189 fecal samples yielded a valid result (the rest were discarded because of contamination by fungi during the growth period). We isolated $M$. avium ssp. paratuberculosis on Herrold's egg yolk medium containing mycobactin $\mathrm{J}$, amphotericin $\mathrm{B}$, and nalidixic acid (Becton Dickinson, Le Pont de Claix, France) according to the method of Whipple et al. (1991). Three tubes of Herrold's medium were read at wk 6, 10, 14 and 16 of incubation at $37^{\circ} \mathrm{C}$. We counted the number of colonies in each tube, and we assigned a semiquantitative value to each fecal sample: "negative" when count $=0$, " + " when count $<20 \mathrm{cfu} /$ tube, " ++ " when count $\geq 20$ and $<100 \mathrm{cfu} /$ tube, and " +++ " when count $\geq 100$ $\mathrm{cfu} /$ tube. For reference, these values corresponded to $1 \mathrm{cfu} / \mathrm{g}, 10$ to $50 \mathrm{cfu} / \mathrm{g}$, and $>50 \mathrm{cfu} / \mathrm{g}$ of feces, respectively.

Detection of MAP Antibodies by Serum. Serum was processed for antibody detection by ELISA. Spe- 
NAVARRO-GONZALEZ ET AL.

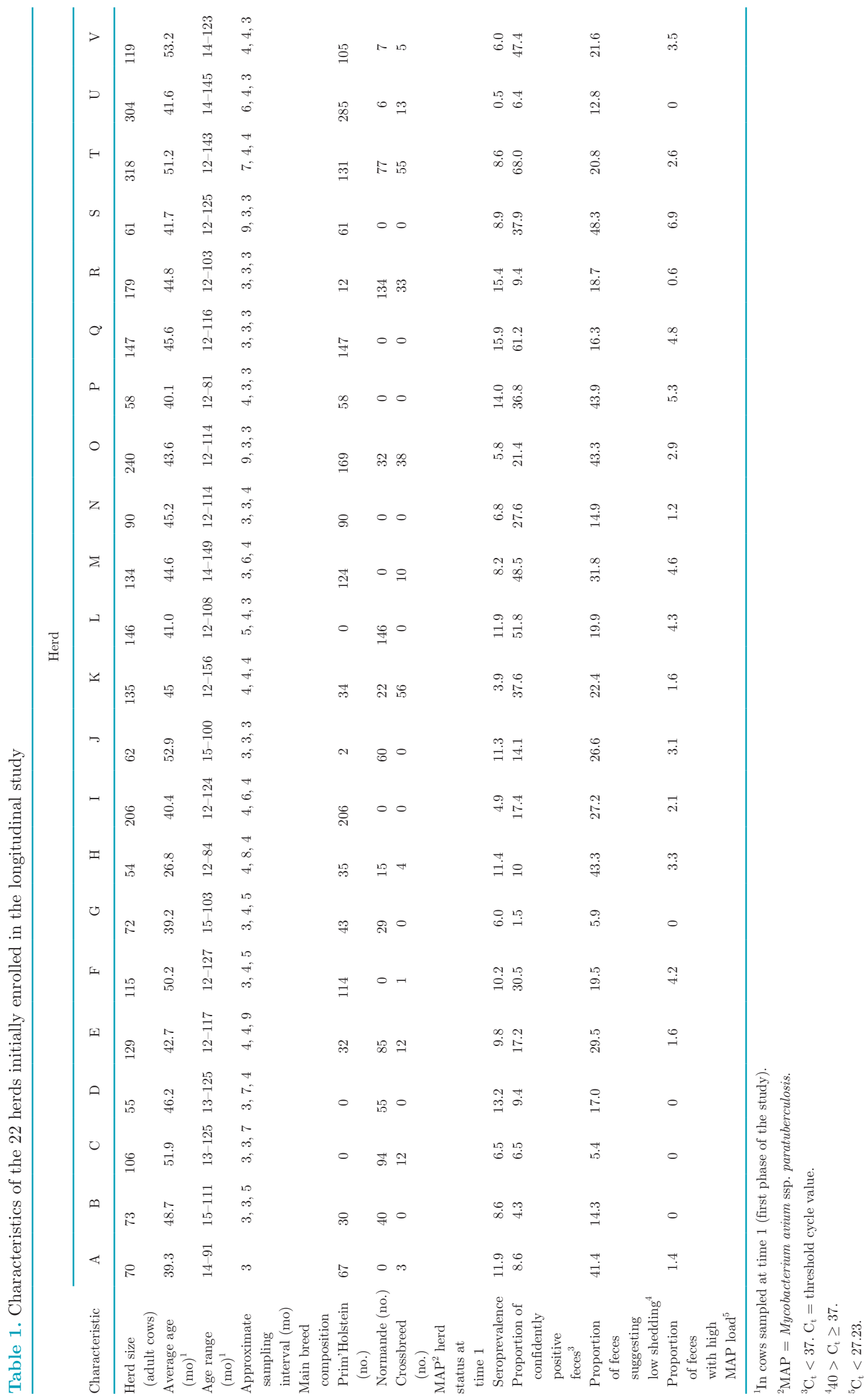


Table 2. Schematic summary of the selection criteria ${ }^{1}$

\begin{tabular}{lcccc}
\hline & \multicolumn{3}{c}{ Proportion of animals (\%) selected to continue based on results at time 1} \\
\cline { 2 - 5 } Animals sampled at time 1 & & & Result of the first quantitative PCR \\
\cline { 2 - 5 } & First ELISA OD & $\mathrm{C}_{\mathrm{t}} \leq 35$ & $\mathrm{C}_{\mathrm{t}}>35$ and $\leq 38$ & $\mathrm{C}_{\mathrm{t}}>38$ or negative \\
\hline All animals older than 12 mo & $<10$ & 100 & 50 & 20 \\
& $\geq 10$ and $\leq 30$ & 100 & 100 & 100 \\
\hline
\end{tabular}

${ }^{1} \mathrm{C}_{\mathrm{t}}=$ threshold cycle; $\mathrm{OD}_{\mathrm{c}}=$ corrected optical density.

cifically, we used the Idexx Paratuberculosis Screening Ab Test (Idexx, Montpellier, France). For each sample, we calculated the sample-to-positive $(\mathrm{S} / \mathrm{P})$ percentage (percentage of the sample optical density to the positive control optical density) and considered it positive if the $\mathrm{S} / \mathrm{P}$ percentage was $\geq 55 \%$, as per the manufacturer's instructions (ftp://s173-183-201-52.ab.hsia.telus.net/ AgroMediaDocs/JDrefs/Pourquier\%20ELISA\%20user \%20guide.pdf).

\section{Preliminary Work for the Use and Interpretation of qPCR Results}

Because $\mathrm{qPCR}$ is an alternative to the reference test (FC) to detect MAP shedding in feces, it was advisable to assess the accuracy of the qPCR against FC. For such a continuous-scaled test such as qPCR, the receiver operating characteristic (ROC) curve is useful, because it plots the sensitivity and 1 - specificity values that result as the test's threshold value is varied; the area under the ROC curve measures its overall diagnostic accuracy (Mandrekar, 2010). However, ROC analysis assumes that the reference test has a perfect sensitivity and specificity (Wang et al., 2006), and MAP FC is known to be imperfect, with a low sensitivity to detecting low shedders in particular, and qPCR methods can perform better in bacterial diagnostics than the tests classically considered to be reference methods (Kralik and Ricchi, 2017). For this reason, we aimed to find a $\mathrm{C}_{t}$ threshold that optimized the accuracy, but also to interpret results in a biologically sensible manner according to current knowledge of MAP. The following sections explain in detail the steps we followed for these procedures. We also explain how we found a $\mathrm{C}_{t}$ threshold that corresponded to high shedding as determined by $\mathrm{FC}$, for a semiquantitative use of $\mathrm{qPCR}$.

Optimization of the qPCR Technique. We performed an ROC curve to find a $\mathrm{C}_{t}$ cutoff value that represented a trade-off of sensitivity and specificity for the qPCR test, with FC as reference test. Of the 1,223 individual fecal samples that were processed for both FC and qPCR to detect MAP fecal shedding,
1,179 provided valid paired results that could be used to build the ROC curve. We implemented the ROC curve with R Software (R Core Team, 2016) package pROC (Robin et al., 2011). The resulting ROC curve (area under the curve 0.76) indicated that a threshold of $\mathrm{C}_{t}=37$ provided a good trade-off of sensitivity and specificity (sensitivity 0.70, specificity 0.70 ) for on-farm control with a test-and-cull strategy. That is, this $\mathrm{C}_{t}$ value maximized the likelihood of culling animals that were truly positive and not false qPCR positives, and to not consider animals as false positives when they were true shedders. Hereafter, we refer to the cutoff of $\mathrm{C}_{\mathrm{t}}<37$ as the "adjusted cutoff value."

Interpretation of $q P C R$ Ct Values Between the Standard and the Adjusted Cutoff. Of 2,658 fecal samples successfully tested by $\mathrm{qPCR}$ at time 1 , 618 had a $\mathrm{C}_{\mathrm{t}}$ value $\geq 37$ and $<40$. This represented almost $23 \%$ of the tested population. For 279 of 618 , a concurrent FC result was available. Of those, 233 were culture-negative but would have been considered qPCR-positive using the standard cutoff value $\left(\mathrm{C}_{t}<\right.$ 40), and we questioned how they related to FC-positive samples in the same $C_{t}$ value range. The $C_{t}$ values were similarly distributed across the $\mathrm{FC}$ semiquantitative categories. Of the 279 samples in the $\mathrm{C}_{\mathrm{t}}$ range $\geq 37$ and $<40$ and with a concurrent FC result, 46 were culture-positive, with a low level of shedding $(\mathrm{FC}+)$ as the most frequent positive result $\left(\mathrm{n}_{+}=40\right)$.

Only about $23 \%$ (276) of the total set of samples tested by FC were MAP-positive using this technique. Thus, these 46 FC-positive samples represented an important amount, and suggest that the $\mathrm{C}_{t}$ range between the adjusted and standard cutoffs has a biological significance that needs to be correctly interpreted. For samples in that $\mathrm{C}_{t}$ range, it is no longer clear whether the ability of qPCR to detect DNA from nonviable cells influences the outcome or whether it corresponds to a low level of shedding that the FC is mostly unable to detect.

In light of the known low sensitivity of FC for low shedders and to preserve the negative predictive value of the qPCR test, we considered samples in the $\mathrm{C}_{t}$ 


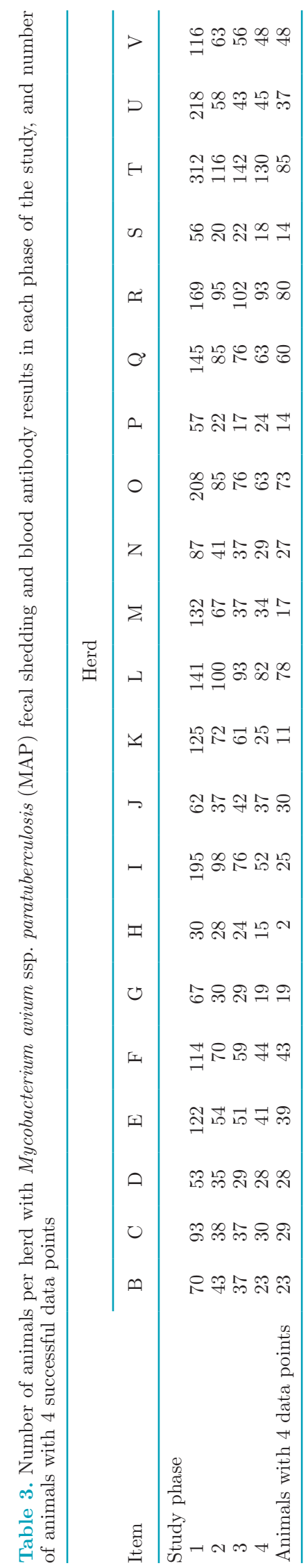

Journal of Dairy Science Vol. 102 No. 10, 2019 range $\geq 37$ and $<40$ as "low or nonculturable" MAPpositives. We referred to those samples with an "*"and have described their contribution to the fecal shedding patterns. We are aware that this qPCR category includes low shedders (truly infected) that cannot be detected by FC and may also include passive shedders (animals that ingest MAP from the environment and shed it in feces with no true tissue infection). However, we believe that the recognition of this qPCR category and the description of its contribution to fecal shedding traits could provide a new view of the variability of MAP infections and the diversity of fecal shedding patterns.

Samples with a $\mathrm{C}_{\mathrm{t}}<37$ are represented with a "+" and referred to as "confidently positive" hereafter. To refer to samples with a $\mathrm{C}_{t}<40$, whether "** or "+," we have used the term "non-negative."

Use of $q P C R$ as Semiquantitative Indicator of Fecal Shedding. To relate fecal shedding to qPCR, we sought to determine the $\mathrm{qPCR}$ cutoff values that best related to the categories of the semiquantitative FC. For this, we used the 192 samples with concordant positive results for both methods. Using a classification tree model, we aimed to identify the splits in the qPCR values that provided the purest groups of $\mathrm{FC}$ categories $(+,++$, and +++$)$. We implemented the classification tree model using R Software (R Core Team, 2016), specifically the "rpart" package (Therneau et al., 2015).

\section{Strategy of Analysis}

Description of Fecal Shedding Traits: Shedding Patterns, Continuity, and Progression. We used animals with 4 valid qPCR results to describe the fecal shedding patterns most accurately. To describe fecal shedding, a fecal sample was considered MAP-negative $(-)$ when $\mathrm{C}_{\mathrm{t}} \geq 40$, confidently positive $(+)$ when $\mathrm{C}_{\mathrm{t}}$ $<37$, and low or nonculturable $(*)$ when $\mathrm{C}_{\mathrm{t}}<40$ and $\geq 37$. With the temporal set of 4 fecal samples per animal, we described the patterns of several fecal shedding traits using different approaches and classification criteria. First, we focused on the non-negative, consecutive sampling events per animal (see specific criteria in Table 4). Hereafter, we refer to these as "fecal shedding patterns." Note that "non-negative" refers here to any sample with $\mathrm{C}_{t}<40$. Second, we were interested in exploring the "continuity of shedding" as defined by Mitchell et al. (2015a). The main criterion for establishing continuity patterns was the continuity of nonnegative sampling events after the first non-negative sample (see Table 5). Finally, we determined the patterns that indicated an evolution toward an increased amount of MAP shed in feces, similar to the patterns first defined by Schukken et al. (2015) ("progression 
patterns"; Table 6). To describe progression, we applied the adjusted qPCR cutoff value to be more restrictive by considering only confidently positive samples. We also applied the semiquantitative threshold for high shedding, thus defining progression patterns based on both confident positivity and evolution toward high fecal shedding.

Description of Serological Patterns. Animals with 4 valid ELISA results were used to describe serological patterns and their distribution within the observed fecal shedding patterns, continuity of shedding, and progression. Serological patterns were defined based on the number of MAP-antibody-positive events determined by serum ELISA (categories A0 to A4, meaning zero positive samples to 4 positive samples).

Individual Cow Information. The age, breed, and parity of the cows were known from the national database SNIG of the Centre de Traitement de l'Information Génétique. The age in months at time 1 was calculated; thereafter, animals that continued the study were classified into age categories (in years) for data analyses. Breeds in the initial study sample were Prim'Holstein (818 individuals), Normande (498 individuals), crossbreeds (122 individuals) and Pie Rouge des Plaines (37 individuals). To explore the effect of parity on fecal shedding and serology, we created the following categories: nulliparous cows, nulliparous cows that became primiparous during the study period, primiparous cows, and multiparous cows. Age at time 1 and parity were treated as categorical ordinal variables.

Data Visualization and Statistical Analysis. We plotted response variables (fecal shedding traits, serological patterns) against the categorical explanatory variables (age, breed, parity) using mosaic plots implemented in R Software (R Core Team, 2016) with the packages ggplot2 (Wickham, 2009) and ggmosaic (Jeppson et al., 2017). Mosaic plots are useful for visualizing categorical data: they consist of a set of rectangles (or "tiles") that represent the cells in a contingency table, and the area of each tile is proportional to its corresponding cell (Hofmann, 2008). We built 2-way contingency tables to plot 1 explanatory variable versus 1 response variable each time, and to test the null hypothesis of independence in these 2-way tables.

To test whether there was a significant departure from independence (that is, an effect of the explanatory variables on the response variables), we aimed to capture deviations from zero in the Pearson residuals (r), a measure of discrepancy between observed and expected values. For this purpose, the statistic we tested was the maximum of the absolute values of Pearson residuals $(\boldsymbol{M})$, because it is "particularly suitable for identifying the cells responsible for the 'dependence'" (Zeileis et al., 2007). Following the instructions from Zeileis

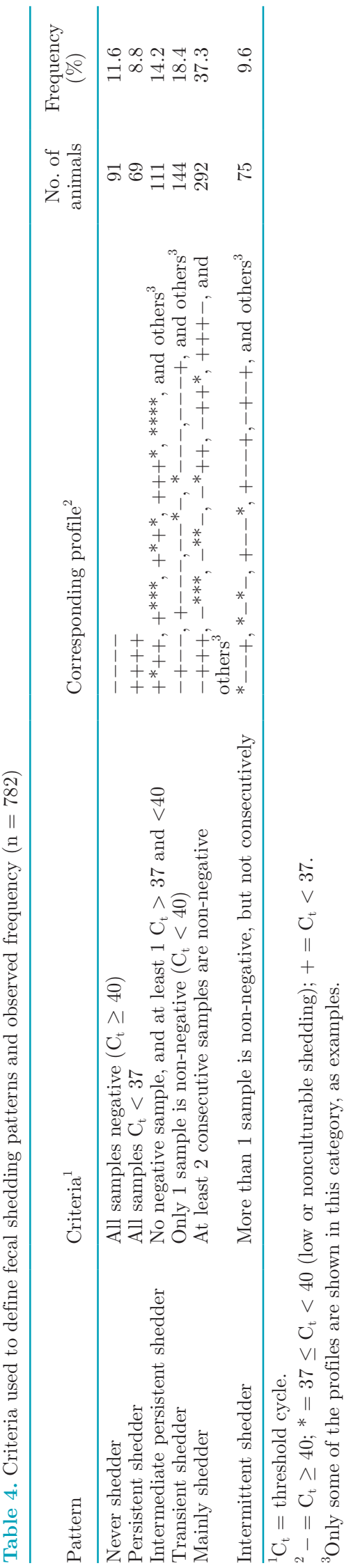

Journal of Dairy Science Vol. 102 No. 10, 2019 
NAVARRO-GONZALEZ ET AL.
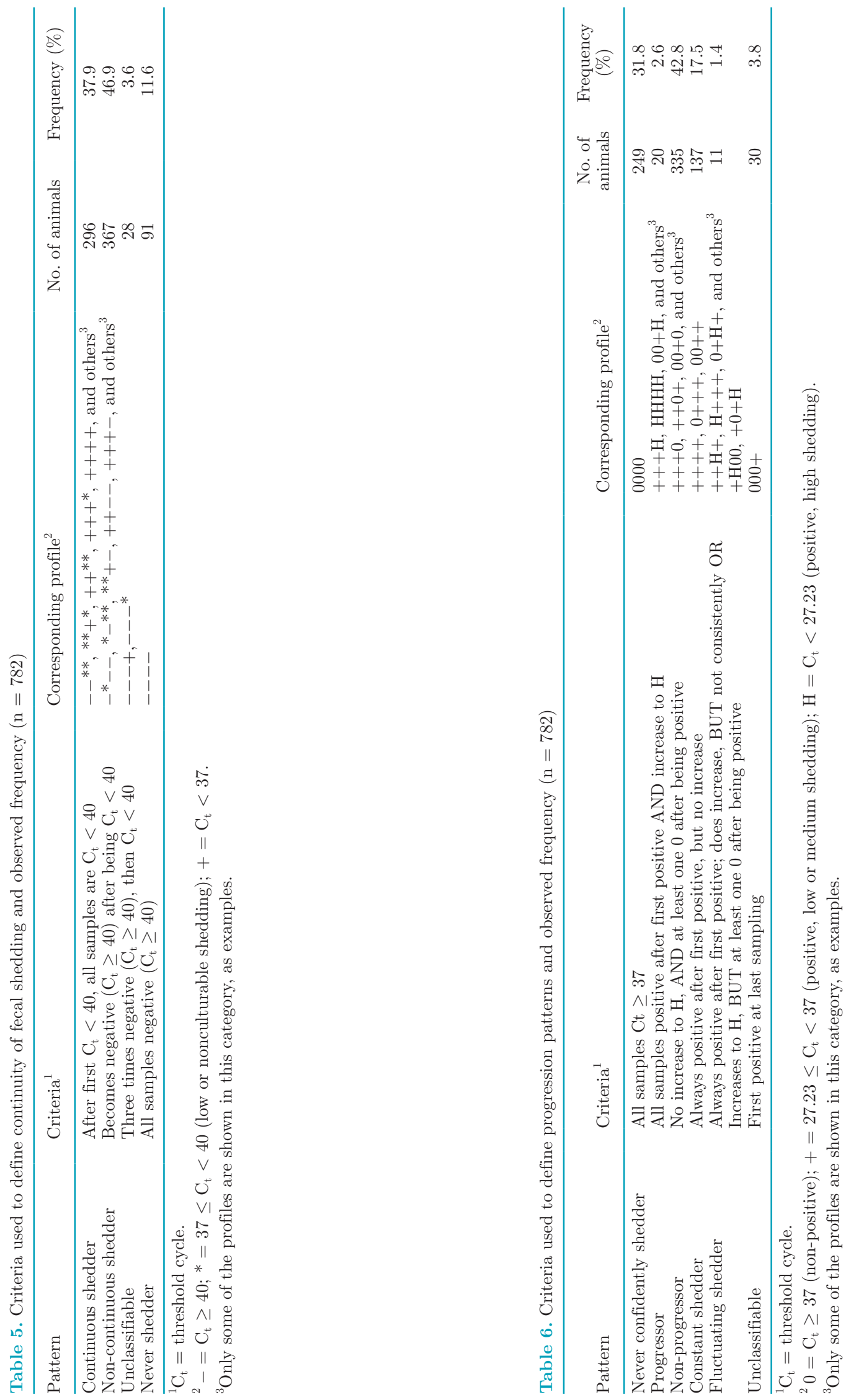
et al. $(2007,2017)$ for each combination of response and explanatory variable, we performed a permutation test of independence of the maximum statistic using the package vcd (Meyer et al., 2016) in R software (R Core Team, 2016). We computed the $P$-value from the permutation distribution under independence generated via sampling 5,000 tables using replacement with the same row and column sums and computing the maximum statistic for each of these tables, with a significance level set at $\alpha=0.05$. We followed the same procedure to test the independence of serological patterns and fecal shedding traits.

In the final study sample, some categories had a small sample size (for example, only 4 and 2 animals were in the age categories 10 and $11 \mathrm{yr}$, respectively). Despite the fact that the permutation test for independence can deal with cell sizes smaller than 5, we decided to remove those animals from the data visualization and the statistical analyses of the effect of age on the response variables. We argued that the herd of origin might play an important role in fecal shedding traits, so we wanted to avoid potential bias due to some herds being represented in these categories with a small sample size. For the same reason, in the analyses of the effect of breed, we removed the 17 animals of the breed Pie Rouge des Plaines. Similarly, only 2 animals from herd $\mathrm{H}$ completed the study (Table 3 ), so they are not shown in the mosaic plots by herd, and this herd was not considered in comparisons between herds.

\section{RESULTS}

\section{Semiquantitative qPCR Categories of MAP Fecal Shedding}

The pruned classification tree had 2 splits and 3 terminal nodes (Figure 1). In brief, the first split $\left(\mathrm{C}_{\mathrm{t}}\right.$ $\geq 31.12)$ predicted low fecal shedding $(+)$ in a terminal node with a $17.9 \%$ misclassification rate and $93.2 \%$ sensitivity. In the next split, the cutoff value $<27.23$ provided the purest classification for high fecal shedding $(+++$; that is, the smallest number of misclassified samples). This node was associated with a misclassification rate of $14.3 \%$ and a sensitivity of $76.4 \%$ (42 of the 55 high shedders in the total set were included in this node).

The terminal node that predicted medium fecal shedding $\left(++, 31.12>\mathrm{C}_{t} \geq 27.23\right)$ performed the worst, with a $53.8 \%$ misclassification rate and a $35.3 \%$ sensitivity. This result may have been due to the small number of medium shedders in the data set (34). In light of the low predictive power for medium fecal shedding, we decided to combine the low and medium shedding categories as follows: low or medium fecal shedding $\left(\mathrm{C}_{\mathrm{t}}\right.$ $\geq 27.23)$ and high fecal shedding $\left(\mathrm{C}_{t}<27.23\right)$. Instead of creating a medium-high shedding category by joining the medium and high shedding terminal nodes, we preferred to preserve the low misclassification rate of the high fecal shedding category. This ensured that most animals classified as high shedders were true high shedders, even though not all high shedders were classified as such.

\section{Proportion of MAP Shedders in the Final Study Sample}

Of the animals tested 4 times, $68.2 \%$ were confidently positive $\left(\mathrm{C}_{t}<37\right)$ at least once during the study period. This number increased to $88.4 \%$ when we included animals that had "** at least once, potentially indicating passive shedders, low or nonculturable MAP shedding in feces, or both. The initial fecal shedding proportion (proportion of animals positively detected as MAP fecal shedders) by herd was known because all animals older than 12 mo were tested by qPCR in feces at time 1 of the study. Although all herds had a high seroprevalence in the previous year, the fecal shedding proportion varied largely between herds, ranging from $1.5 \%$ (herd G) to $61.2 \%$ (herd Q; Table 1). This table also shows the percentage of animals with a $C_{t}$ value between the adjusted and standard cutoffs at time 1: that is, the percentage of low or nonculturable samples, which varied largely between herds (minimum $5.4 \%$ and maximum 43.9\%). In 13 herds, the proportion of low or nonculturable samples was larger than the proportion of samples with $\mathrm{C}_{\mathrm{t}}<37$ (confidently positive).

\section{Description of Fecal Shedding Patterns}

Fecal Shedding Patterns in the Final Study Sample. We found 80 different permutations of qPCR categories $(+, *$, or -$)$ in this longitudinal assessment of the 782 animals that were successfully followed up 4 times. We classified these qPCR profiles into 6 fecal shedding patterns, with frequencies, as shown in Table 4. Briefly, those fecal shedding patterns were never shedder (4 times negative), persistent shedder (4 times confidently positive), intermediate persistent shedder (no sample negative, and 1 to 3 samples confidently positive), transient shedder (3 times negative, 1 time non-negative), mainly shedder (non-negative at least 2 times consecutively) and intermittent shedder (nonnegative more than 1 time, but not consecutively). The most frequently observed fecal shedding pattern was mainly shedder $(37.3 \%)$; the rest were variably distributed into the other patterns.

In all herds that participated in the 4 stages of the study ( 21 herds), some animals were negative by qPCR 


\section{Pruned Classification Tree of FC vs qPCR}

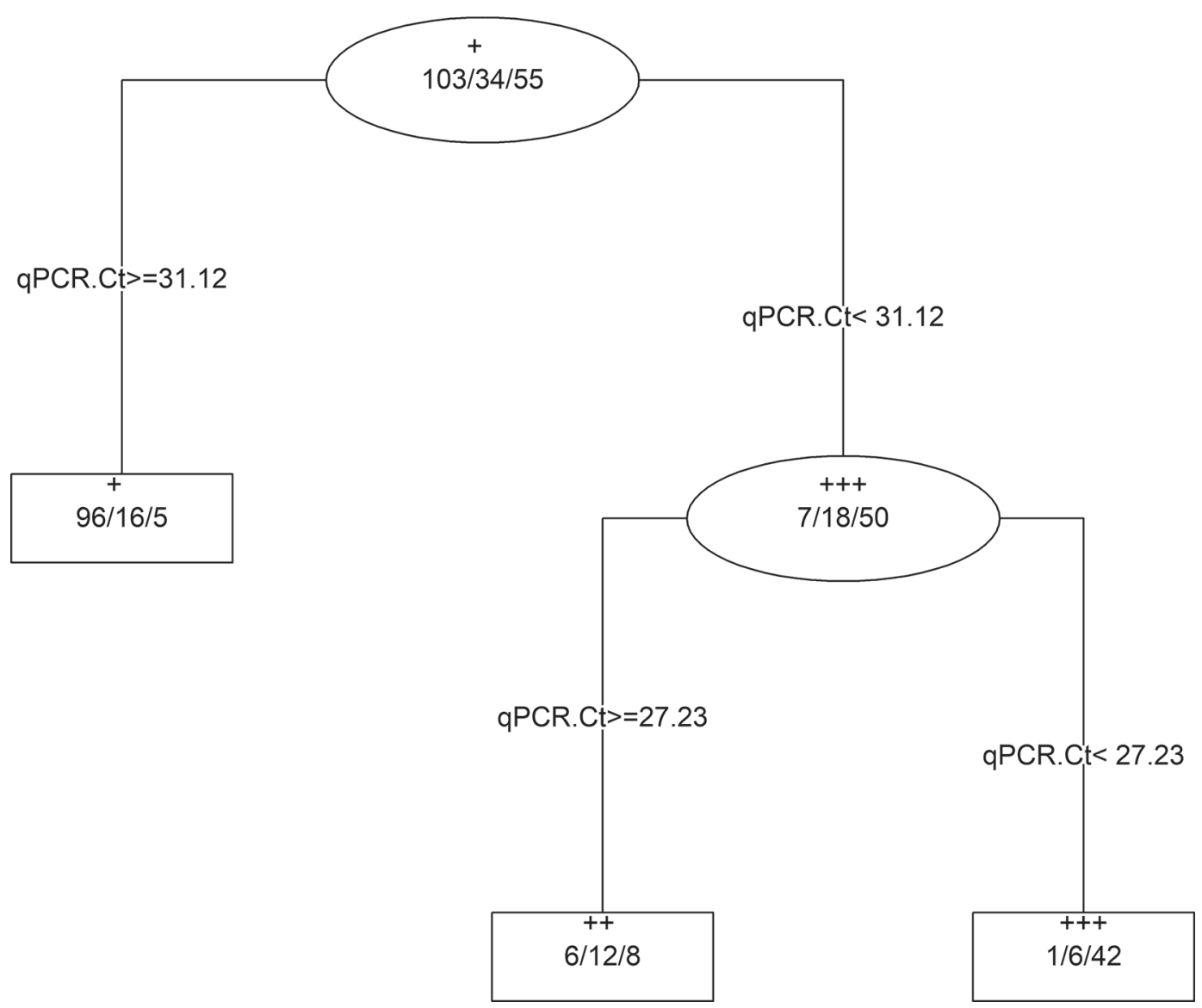

Figure 1. Graphic outcome of the classification tree model implemented to find the splits in the quantitative (q)PCR values that best related to the paired semiquantitative results obtained by fecal culture (FC). Numbers inside the shapes refer to the number of samples classified as + , ++ , and +++ , respectively, in a specific node. Above these numbers, the predicted category for each node is shown. Classification thresholds are shown in the corresponding branches, and rectangles indicate terminal nodes. Only the cycle threshold $(\mathrm{Ct})<27.23$ was used to differentiate high shedders and low to medium shedders.

at time 1 [specifically, 363 cows of $782(46.42 \%)]$. The majority of these cows (approximately 75\%) started shedding MAP in feces at some point during the following 9 to $13 \mathrm{mo}$. At the first sampling event, 419 cows were non-negative for MAP fecal shedding assessed by qPCR. Of those, $57.04 \%$ transitioned to negativity $\left(\mathrm{C}_{\mathrm{t}}\right.$ $>40$ ) at some point during the study period.

Fecal Shedding Patterns by Herd. Figure 2 shows the proportion of fecal shedding patterns by herd. Herds are kept in the same order in the mosaic plot showing continuity of shedding, progression, and serological patterns to enable direct comparisons.

We found all 6 fecal shedding patterns in 8 of the 20 herds. Each herd population was fragmented into at least 4 patterns, with the exception of 1 herd (B), in which we detected only 3 patterns. In 9 herds, all of the animals surveyed 4 times shed at least once during the study period. Persistent shedders were present in 14 herds, and in 8 of those they coexisted with never shedders. We defined 3 main herd types (Figure 2). First, we observed a type of herd in which never shedders were absent (e.g., Q, R, T, or V). In these herds, the majority of animals were shedding more than once (see the accumulated proportion of persistent shedders, intermediate persistent shedders, mainly shedders, and intermittent shedders). Conversely, we observed a type of herd in which persistent shedders were absent (e.g., $\mathrm{B}, \mathrm{G}$, or $\mathrm{U}$ ) and a considerable abundance of never shedders $(>37 \%)$. However, the abundance of never shedders was not fully associated with the absence 
of persistent shedders. We found other herds with a similar proportion of never shedders (e.g., herd C) that did have a small proportion of persistent shedders. In

\section{Shedding Patterns}

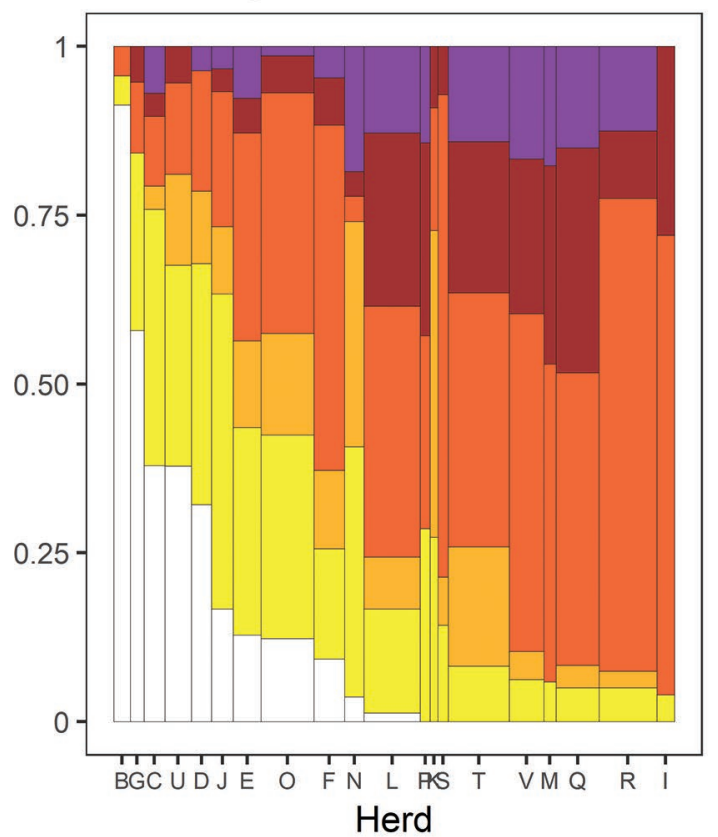

Continuity Patterns

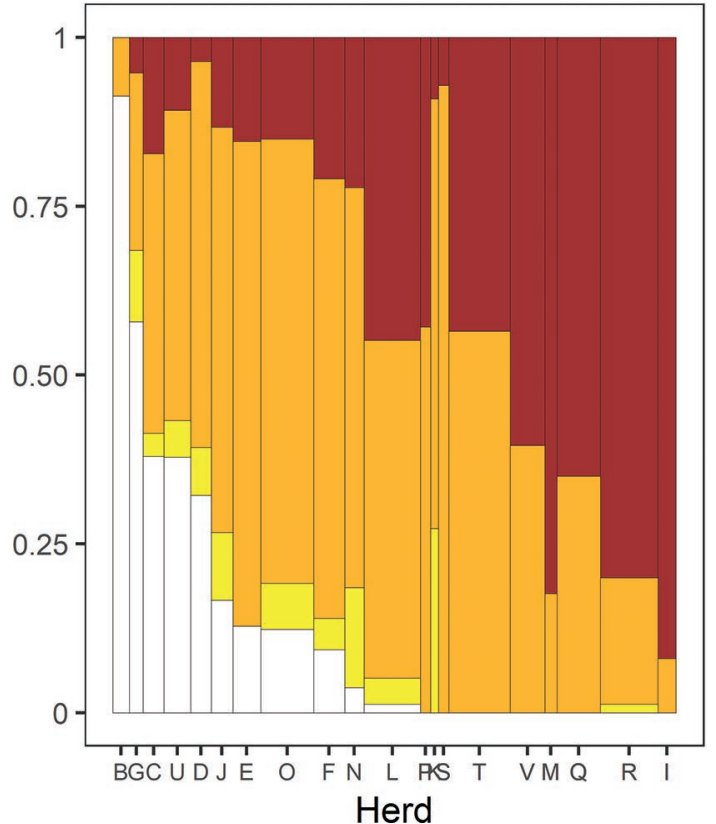

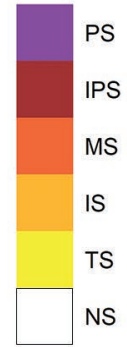

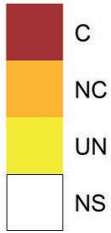

the third type of herd, the population was highly fragmented into the different shedding patterns (e.g., L or $\mathrm{O})$.

\section{Progression Patterns}

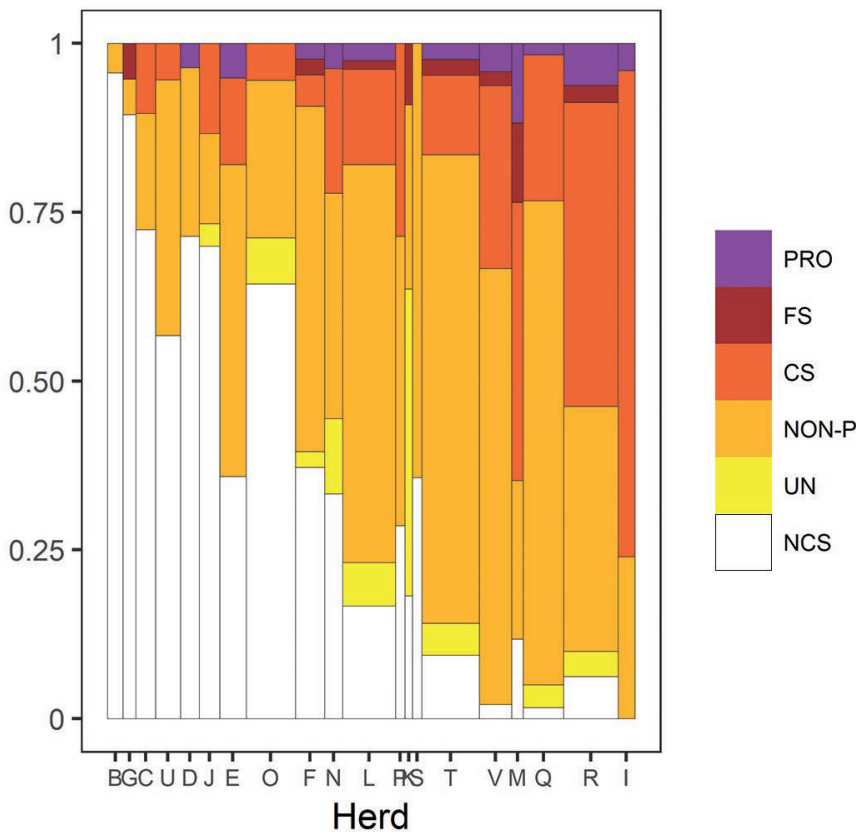

Serological Patterns

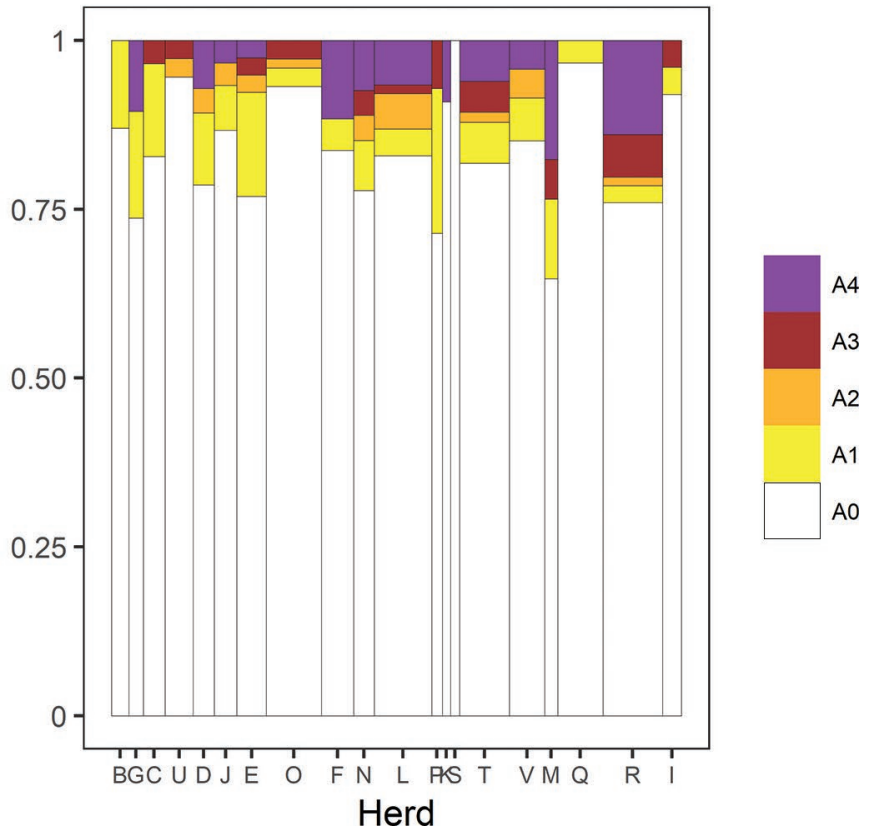

Figure 2. Mosaic plots showing the distribution of fecal shedding traits and serological patterns by herd. For column width reference, herd $\mathrm{K}$ was represented by 11 animals and herd T by 85 animals. In the plot, herds are ordered by descending percentage of "never shedders." Herds without "never shedders" are shown in ascending order of percentage of animals shedding consecutively at least twice. NS = non-shedder; TS $=$ transient shedder; IS = intermittent shedder; MS = mainly shedder; IPS = intermediate persistent shedder; PS = persistent shedder; UN $=$ unclassifiable; $\mathrm{NC}=$ non-continuous; $\mathrm{C}=$ continuous; $\mathrm{NCS}=$ never confidently shedder; $\mathrm{NON}-\mathrm{P}=$ non-progressor; $\mathrm{CS}=$ constant shedder; $\mathrm{FS}=$ fluctuating shedder; $\mathrm{PRO}=$ progressor; $\mathrm{A} 0=$ never positive; $\mathrm{A} 1=$ positive 1 time; A2 = positive 2 times; A3 = positive 3 times; A4 = always positive. 
Fecal Shedding Patterns by Animal Characteristics. Visualization of the distribution of fecal shedding patterns among age categories (in years) at first sampling did not suggest a clear trend toward any shedding pattern as age increased (Figure 3 ). The permutation test for independence was significant $(P=$ $0.03, M=3.27$ ), but only 1 cell was responsible for this effect: the percentage of intermediate persistent shedders was higher at age 1 yr than afterward. Neither the association of fecal shedding patterns with the main breeds nor the association with parity category was significant (Figure 4, $P=0.09, M=2.16$; and Figure 5, $P=0.27, M=2.03)$.

\section{Description of Continuity of Fecal Shedding}

Continuity of Fecal Shedding in the Final Study Sample. With respect to continuity of shedding, $20.84 \%$ of the animals showed no switch in $\mathrm{C}_{\mathrm{t}}$ category (that is,,,-+ or ${ }^{*}$ at all times), but the rest switched categories at least once over the 4 sampling events. Of the 782 animals, 179 (22.9\%) experienced all 3 possible statuses over the 4 sampling events. Of the 295 cows that had a $\mathrm{C}_{\mathrm{t}}<40$ at time 1 and time 2 , only 36 were qPCR-negative at time 3 and time 4 (12.2\%).

Following the definition of continuity of fecal shedding established by (Mitchell et al., 2015a), we classified the qPCR profiles as shown in Table 5. The observed continuity patterns were continuous shedder (all samples were non-negative after the first non-negative), noncontinuous shedder (after being non-negative, at least 1 sample is negative), unclassifiable (non-negative for the first time at the last sampling event) and never shedder (4 times negative). We have reported the frequency of each continuity pattern in Table 5 . Non-continuous shedders were the most frequently observed pattern (46.9\%), followed by continuous shedders (37.9\%).

Continuity of Fecal Shedding by Herd. Roughly, the visualization of continuity patterns by herd showed that the percentage of continuous shedders increased as the percentage of never shedders decreased. Continuous shedders were absent in 1 herd only (herd B; Figure 2 ). The same herd also had the largest proportion of never shedders. The continuity results were important because they provided further insight into the fecal shedding dynamics in herds $\mathrm{S}$ and $\mathrm{K}$, in which a large proportion of animals were shedding more than once consecutively but reverted to qPCR negativity; that is, they were non-continuous shedders.

Continuity of Fecal Shedding by Animal Characteristics. The visualization of continuity of fecal shedding by age group did not suggest any clear indication that continuity was more frequent in older animals. Only 1 cell was responsible for the significant independence of age and continuity: the percentage of unclassifiable continuity at age 8 yr was higher $(P=$ $0.045 ; M=3.05$ ). Note that the "unclassifiable" continuity refers to animals that were qPCR-negative 3 times and became qPCR-positive $(+$ or $*)$ at the last sampling event.

Breed and parity category did not have a significant effect on continuity (Figure $4, P=0.07, M=2.17$, and Figure $5, P=0.17, M=1.98$, respectively).

\section{Description of Progression Patterns}

Progression in the Final Study Sample. In addition to the fecal shedding patterns and the continuity of fecal shedding, we were interested in characterizing the pattern of transition from low or medium shedding to high shedding. We considered disease progressors to be animals that were consistently positive after the first positive result and whose $\mathrm{C}_{t}$ value decreased to the category indicative of high fecal shedding $\left(\mathrm{C}_{t}<\right.$ 27.23 , as found by the classification tree model). This approach was similar to that used by Schukken et al. (2015), although the authors of that study used the counts (cfu) obtained by bacterial culture as an indicator of bacterial load.

Based on the $\mathrm{C}_{\mathrm{t}}$ threshold corresponding to a high level of shedding detected by FC, animals were assigned a "0" (non-positive, $\mathrm{C}_{\mathrm{t}} \geq 37$ ), "+" (low and medium shedding, $27.23 \leq \mathrm{C}_{\mathrm{t}}<37$ ) or " $\mathrm{H}$ " (high shedding, $\mathrm{C}_{\mathrm{t}}<$ $27.23)$ in each of their sampling events. We determined the profile resulting from the 4 sampling events, and thereafter we classified animals according to the criteria in Table 6 to assess their progression status. The main progression patterns and their definition criteria were never confidently shedder (no sample was confidently positive), progressor (all samples were positive after the first positive, and high shedding increased), non-progressor (did not increase to high shedding, and reverted back at least once from the confidently positive category), constant shedder (always confidently positive after the first confidently positive sample, but no increase to high shedding). Less informative progression patterns were the fluctuating shedder (increased to high shedding but reverted back from this category) and unclassifiable (first confidently positive sample at the last sampling event). Table 6 shows the frequency of each progression pattern. Only 1 animal was nonpositive after having a high shedding sample, and only 1 animal transitioned from non-positivity to high-level shedding in just 1 sampling interval.

Progression by Herd. At the herd level, the proportion of high shedders was low (Table 1), ranging from 0 to $6.9 \%$. All herds except herd I had never confidently shedders (Figure 2). Eleven herds had pro- 
gressors in them, and in all cases they coexisted with non-progressors.

Progression by Animal Characteristics. The proportions of progression patterns by age categories

\section{Shedding Patterns}

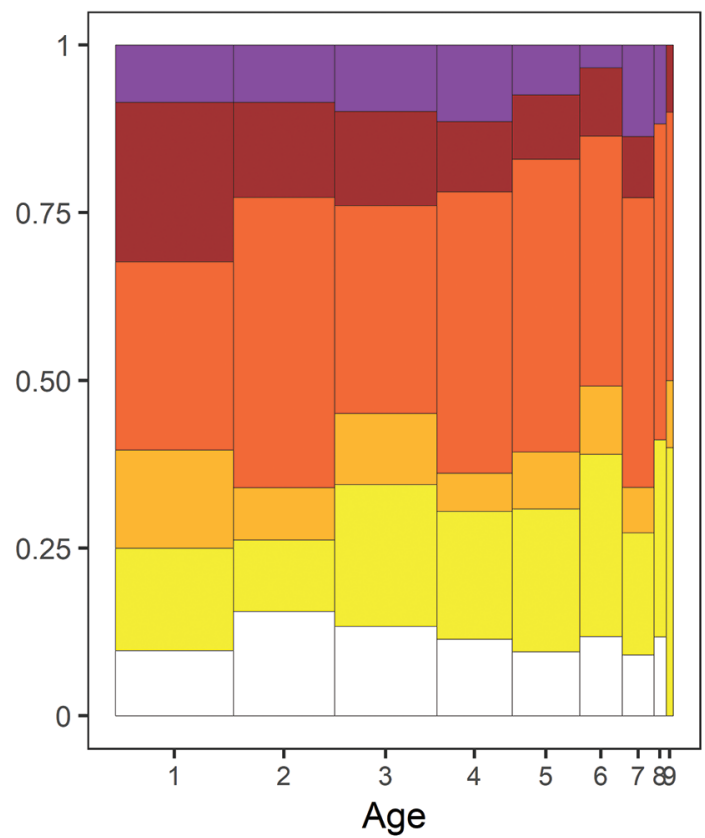

\section{Continuity Patterns}

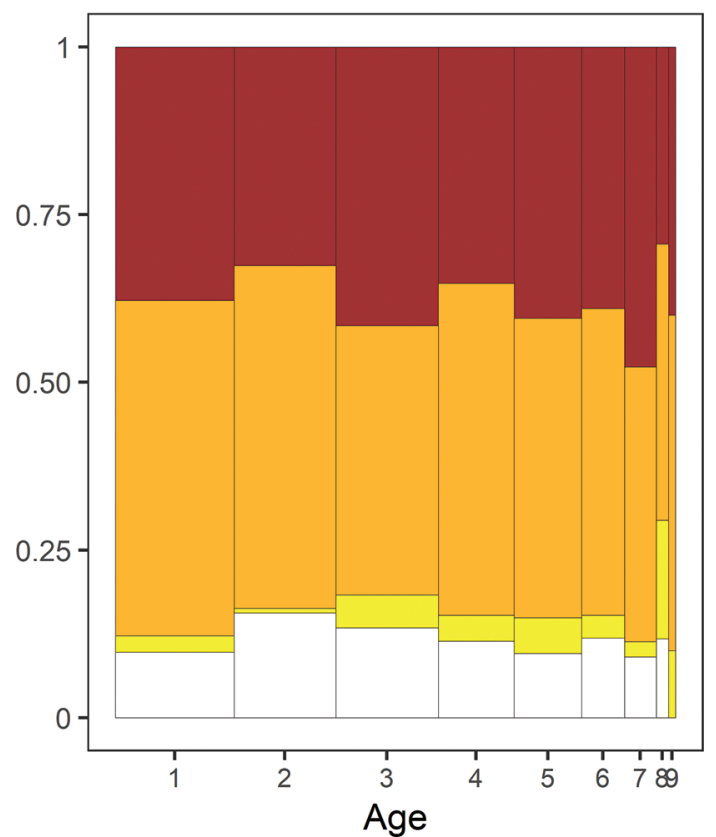

and breed were not significantly different (Figure $3, P$ $=0.24, M=2.64$, and Figure $4, P=0.12, M=2.15$, respectively). However, we found an effect of the parity category (Figure $5, P=0.03, M=2.95$ ), with only

\section{Progression Patterns}

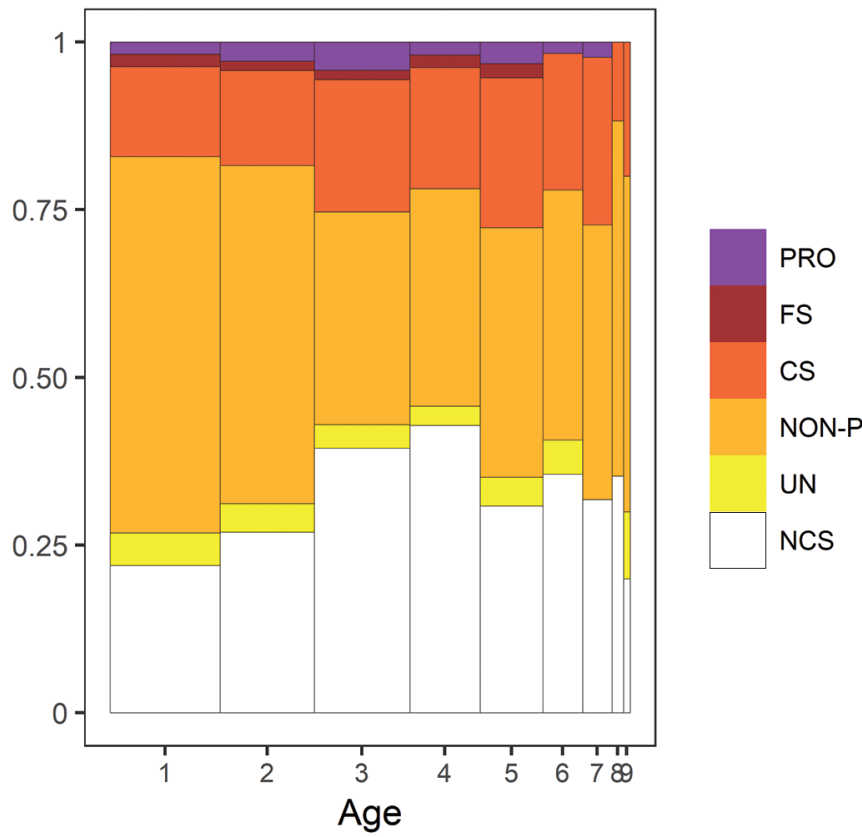

\section{Serological Patterns}

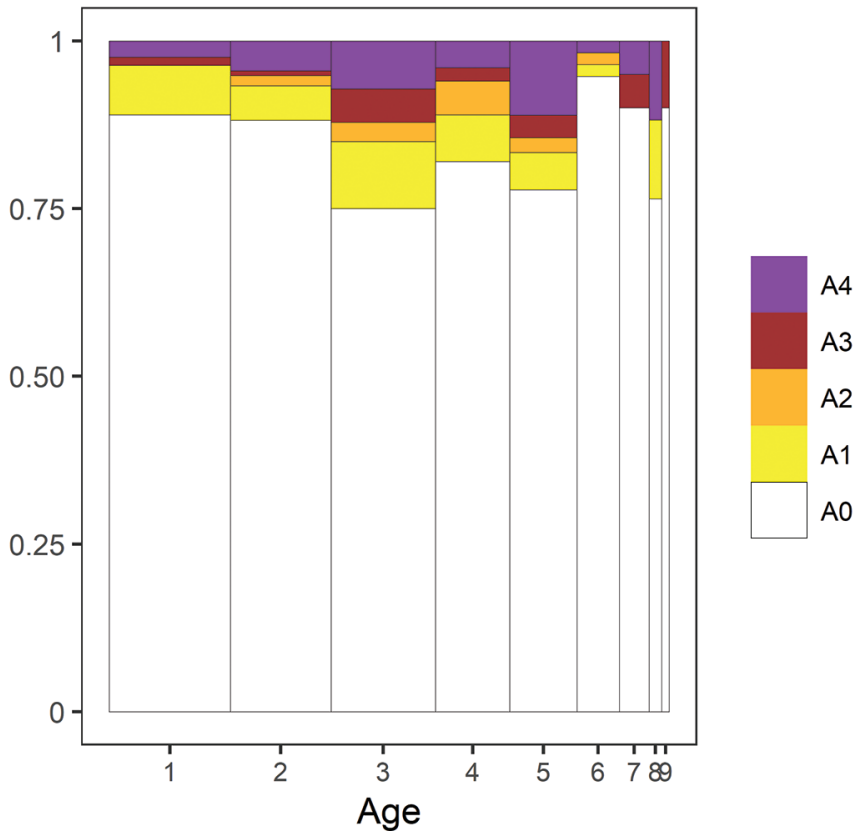

Figure 3. Mosaic plots showing the distribution of fecal shedding traits and serological patterns by age (years) category. NS = non-shedder; TS = transient shedder; IS = intermittent shedder; MS = mainly shedder; IPS = intermediate persistent shedder; PS = persistent shedder; UN $=$ unclassifiable; $\mathrm{NC}=$ non-continuous; $\mathrm{C}=$ continuous; $\mathrm{NCS}=$ never confidently shedder; $\mathrm{NON}-\mathrm{P}=$ non-progressor; $\mathrm{CS}=$ constant shedder; $\mathrm{FS}=$ fluctuating shedder; $\mathrm{PRO}=$ progressor; $\mathrm{A} 0=$ never positive; $\mathrm{A} 1=$ positive 1 time; A2 = positive 2 times; A3 = positive 3 times; A4 = always positive. 


\section{Shedding Patterns}

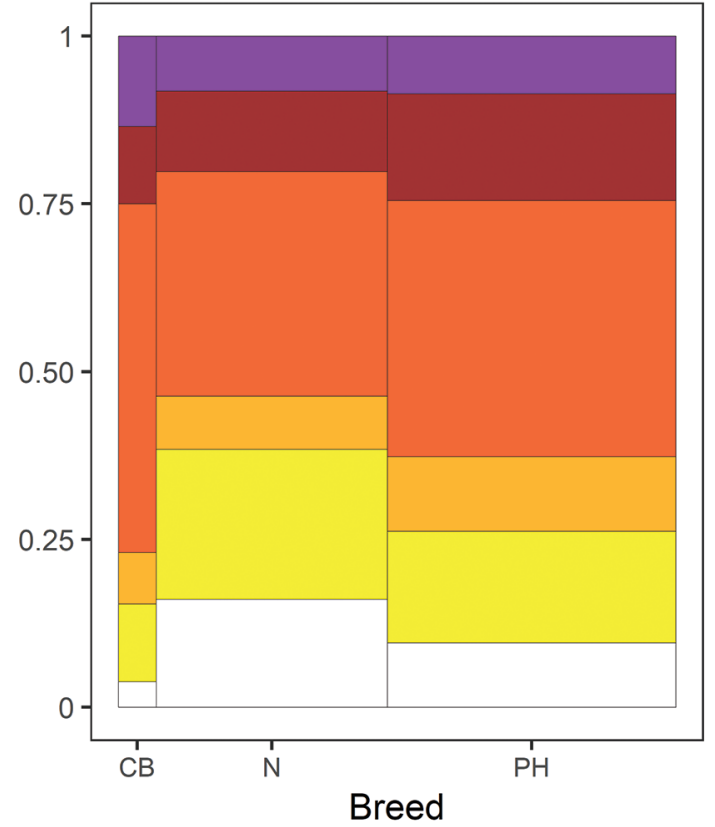

Continuity Patterns

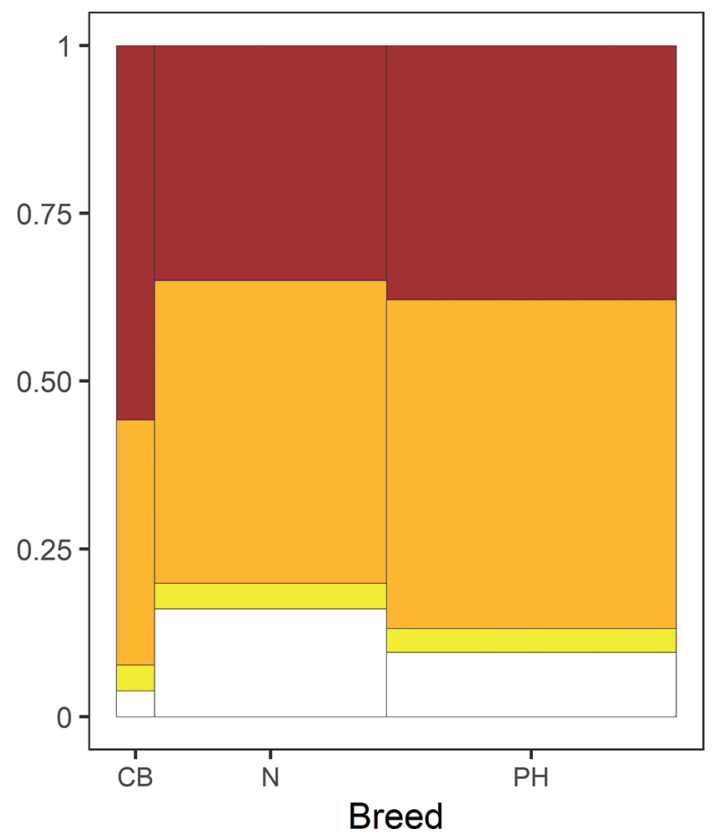

Progression Patterns

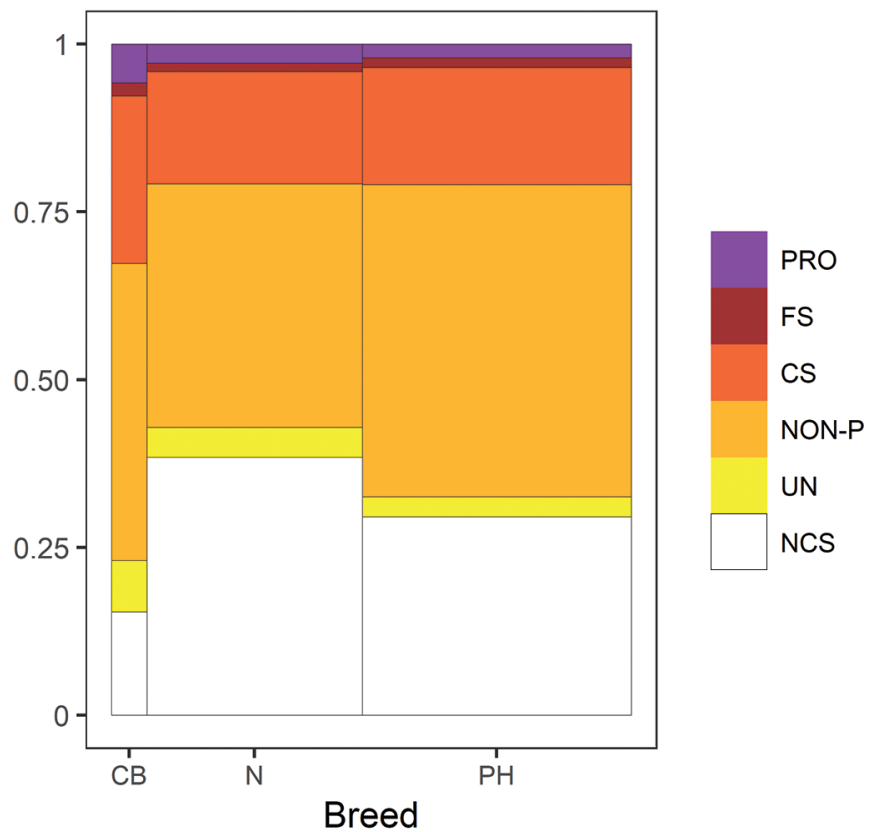

Serological Patterns

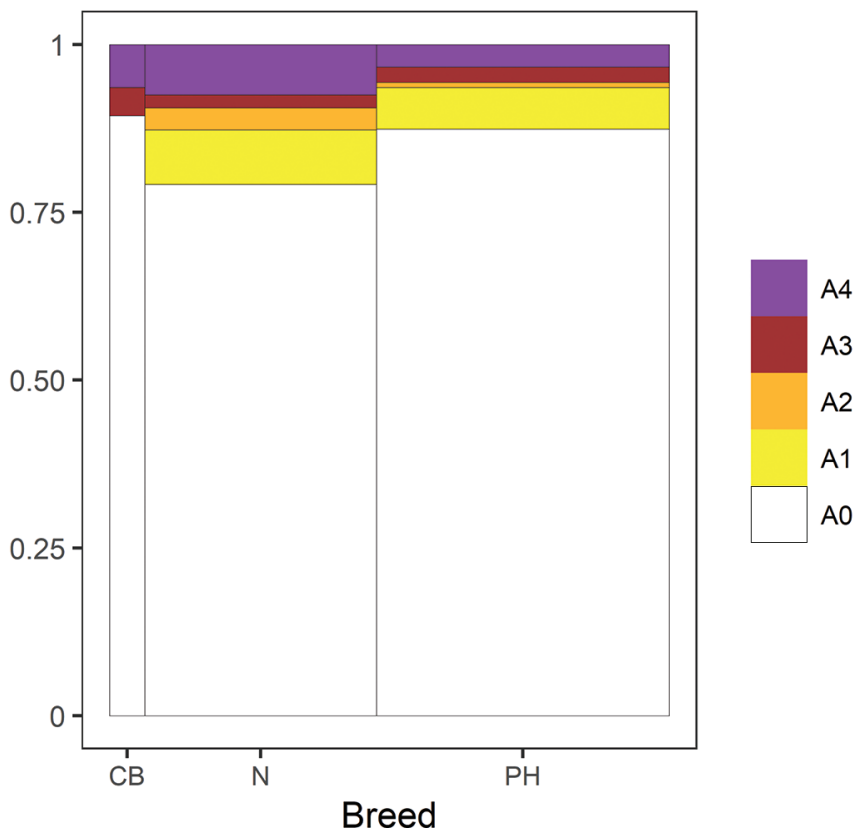

Figure 4. Mosaic plots showing the distribution of fecal shedding traits and serological patterns by breed. CB = crossbreed; $\mathrm{N}=\mathrm{Normande}$; $\mathrm{PH}=$ Prim'Holstein. NS = non-shedder; TS = transient shedder; IS = intermittent shedder; MS = mainly shedder; IPS = intermediate persistent shedder; $\mathrm{PS}=$ persistent shedder; $\mathrm{UN}=$ unclassifiable; $\mathrm{NC}=$ non-continuous; $\mathrm{C}=$ continuous; $\mathrm{NCS}=$ never confidently shedder; $\mathrm{NON}-\mathrm{P}=$ non-progressor; $\mathrm{CS}=$ constant shedder; FS = fluctuating shedder; $\mathrm{PRO}=$ progressor; $\mathrm{A} 0=$ never positive; $\mathrm{A} 1=$ positive 1 time; $\mathrm{A} 2=$ positive 2 times; A $3=$ positive 3 times; $\mathrm{A} 4=$ always positive. 


\section{Shedding Patterns}

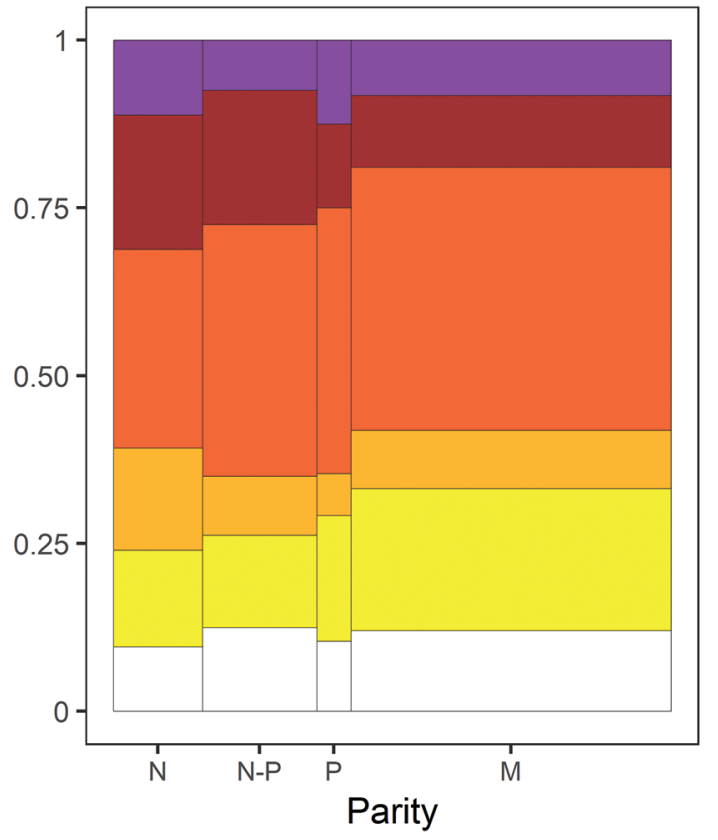

Continuity Patterns

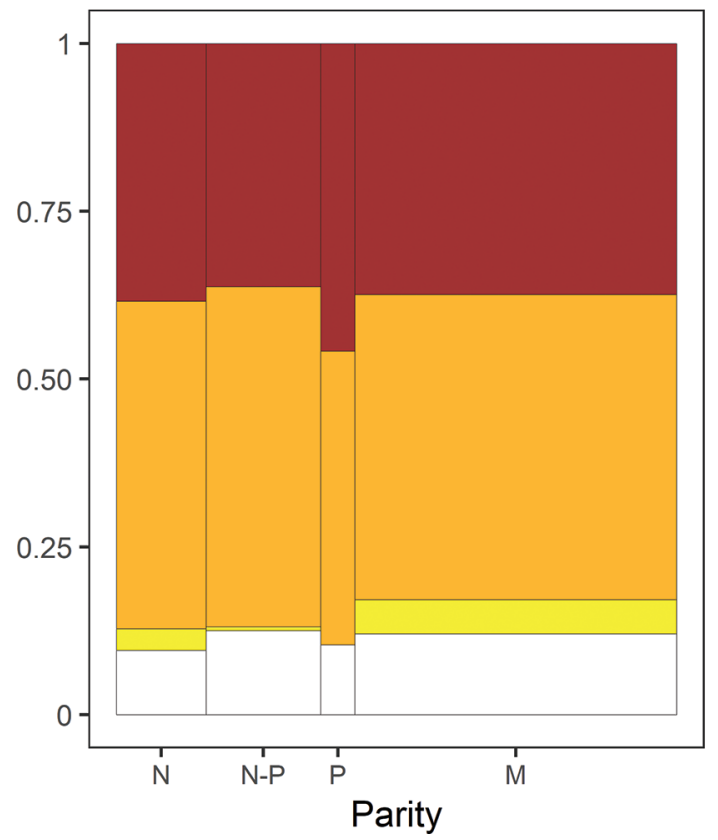

Progression Patterns

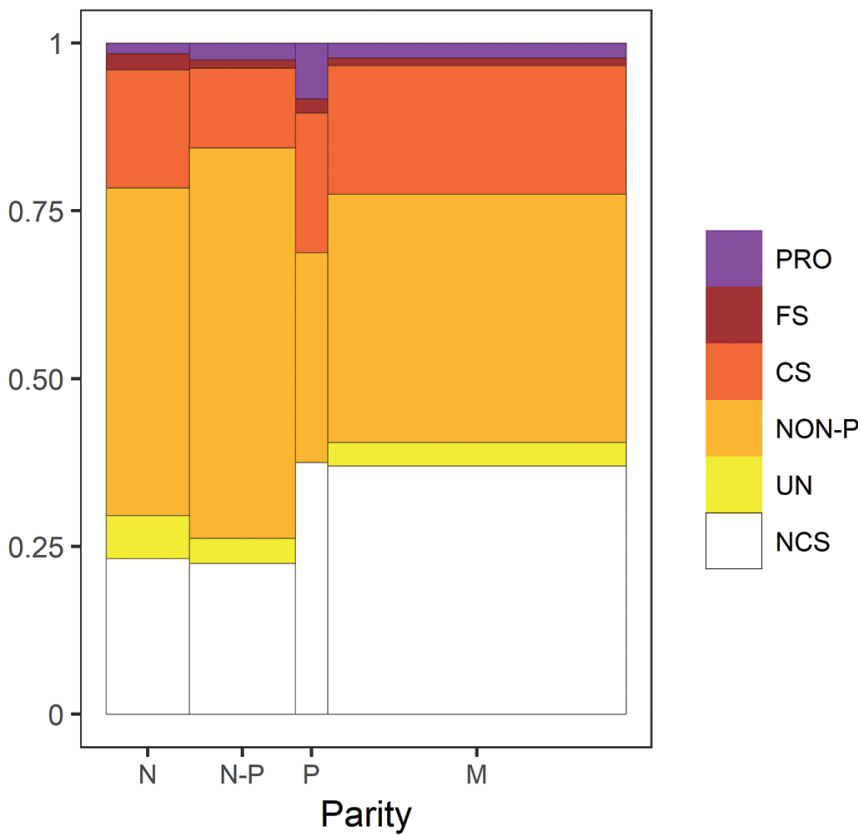

Serological Patterns

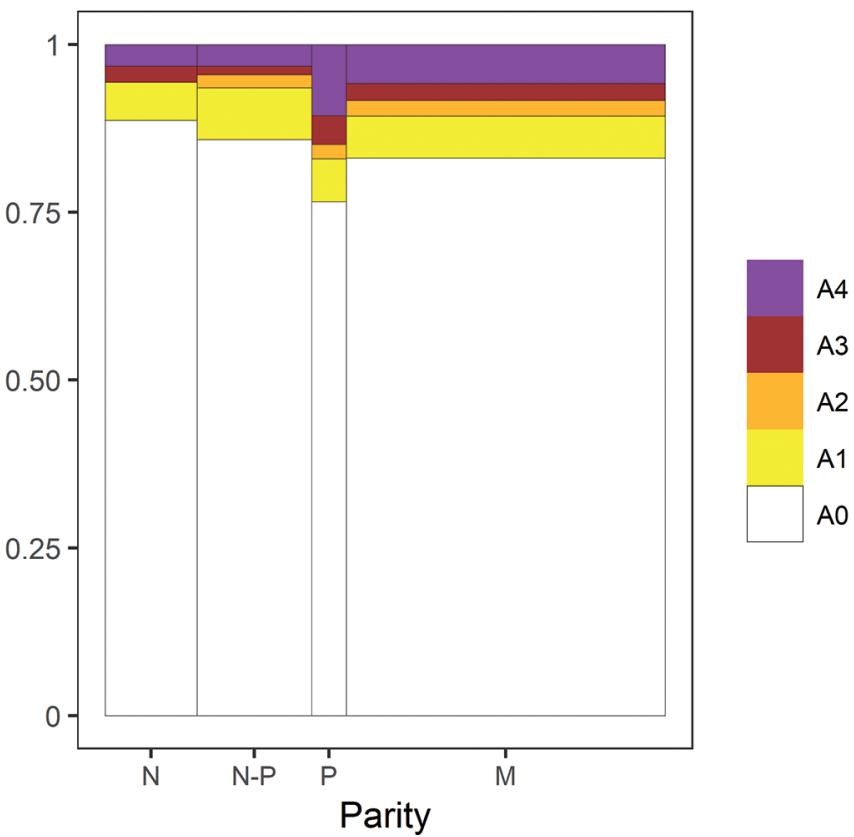

Figure 5. Mosaic plots showing the distribution of fecal shedding traits and serological patterns by parity category. $\mathrm{N}=$ nulliparous; N-P $=$ cows that started as nulliparous and transitioned to primiparous during the study period; $\mathrm{P}=$ primiparous; $\mathrm{M}=$ multiparous. NS $=$ nonshedder; TS = transient shedder; IS = intermittent shedder; MS = mainly shedder; IPS = intermediate persistent shedder; PS $=$ persistent shedder: UN = unclassifiable; $\mathrm{NC}=$ non-continuous; $\mathrm{C}=$ continuous; $\mathrm{NCS}=$ never confidently shedder; $\mathrm{NON-P}=$ non-progressor; $\mathrm{CS}=$ constant shedder; $\mathrm{FS}=$ fluctuating shedder; $\mathrm{PRO}=$ progressor; $\mathrm{A} 0=$ never positive; $\mathrm{A} 1=$ positive 1 time; $\mathrm{A} 2=$ positive 2 times; $\mathrm{A} 3=$ positive 3 times; A4 = always positive. 
Table 7. Distribution of serological patterns by fecal shedding pattern $(\mathrm{n}=757)^{1}$

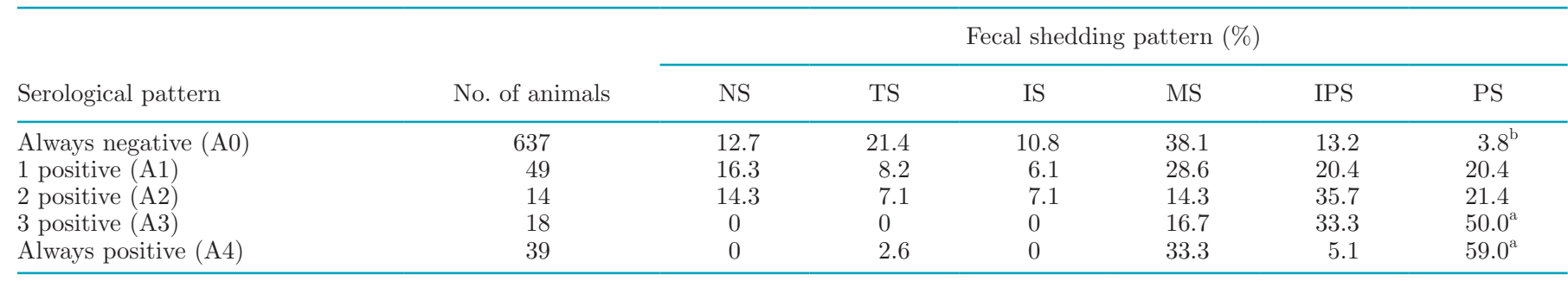

${ }^{\mathrm{a} P}<0.05$, serological pattern positively associated with fecal shedding pattern.

${ }^{\mathrm{b}} P<0.05$, serological pattern negatively associated with fecal shedding pattern.

${ }^{1} \mathrm{IPS}=$ intermediate persistent shedder; IS = intermittent shedder; MS = mainly shedder; NS = non-shedder; PS = persistent shedder; TS = transient shedder.

1 cell significant: the percentage of non-progressors in the group of cows that started as nulliparous and transitioned to primiparous during the study period was higher than in other parity categories. As a reminder, non-progressors were animals that were infected but did not transition to high fecal shedding, and reverted to negativity after being positive.

\section{Serology Results and Their Relation to Fecal Shedding}

We obtained a full set of results (4 qPCR values and 4 ELISA values) for 757 animals. Table 7 shows the definition of the ELISA patterns and the number of animals corresponding to each. The majority of the animals $(84.1 \%)$ showed a serology pattern that was "always negative" (A0). This pattern was present in a large proportion in all herds and ages. Then, 6.5 and $1.8 \%$ of animals were antibody-positive 1 time (A1) and 2 times (A2), respectively. Patterns A3 and A4 were found in 2.4 and $5.2 \%$ of the animals, respectively.

Figure 2 shows the uniformity of distribution of serology patterns among herds, conversely to the distribution of the fecal shedding traits. The serological patterns were independent of age (Figure $3, P=0.37, M$
$=2.47$ ), breed (Figure $4, P=0.15, M=2$ ), and parity category (Figure $5, P=0.62, M=1.66$ ).

Table 7 shows the distribution of serological patterns as a function of the fecal shedding pattern. The permutation test for independence showed a significant association between fecal shedding patterns and serological patterns $\left(P<2.2 \times 10^{-16}, M=10.31\right)$. In persistent shedders, the proportions of serological patterns A4 and A3 were higher $(\mathrm{r}=10.31$ and $\mathrm{r}=5.75$, respectively $)$ and the proportion of A0 was lower $(\mathrm{r}=-4.47)$.

The distribution of serological patterns by continuity patterns is shown in Table 8. Their association was significant $(P=0.003, M=10.31)$; in particular, the patterns A3 and A4 were more frequent in the group of continuous shedders $(r=3.90$ and $r=4.47$, respectively).

We found a significant dependence of serological patterns and progression patterns $\left(P<2.2 \times 10^{-16}, M\right.$ $=11.79)$; their distribution is shown in Table 9. The serological pattern A4 was more frequent in progressors $(\mathrm{r}=11.79)$ and fluctuating shedders $(\mathrm{r}=8.54)$ : that is, categories that implied high shedding. The pattern A3 was more frequent among constant shedders $(\mathrm{r}=3.76)$ : that is, always shedding but no transition to high shedding. The pattern A0 was significantly less frequent in the group of progressors $(r=-3.86)$.

Table 8. Distribution of serological patterns by continuity of fecal shedding $(\mathrm{n}=757)^{1}$

\begin{tabular}{lccccc}
\hline & & \multicolumn{4}{c}{ Continuity pattern (\%) } \\
\cline { 3 - 5 } Serological pattern & No. of animals & NS & UN & NC & C \\
\hline Always negative (A0) & 637 & 12.7 & 4.1 & 51.0 & 32.2 \\
1 positive (A1) & 49 & 16.3 & 2.0 & 30.6 & 51.0 \\
2 positive (A2) & 14 & 14.3 & 7.1 & 21.4 & 57.1 \\
3 positive (A3) & 18 & 0 & 0 & 5.6 & $94.4^{\mathrm{a}}$ \\
Always positive (A4) & 39 & 0 & 0 & 17.9 & $82.1^{\mathrm{a}}$ \\
\hline${ }^{\mathrm{a}} P<0.05$, serological pattern positively associated with continuity pattern. & & \\
${ }^{1} \mathrm{C}=$ continuous; NC = non-continuous; NS = non-shedder; UN = unclassifiable. & &
\end{tabular}


Table 9. Distribution of serological patterns by progression of fecal shedding $(\mathrm{n}=757)^{1}$

\begin{tabular}{|c|c|c|c|c|c|c|c|}
\hline Serological pattern & No. of animals & \multicolumn{6}{|c|}{ Progression pattern $(\%)$} \\
\hline Always negative (A0) & 637 & 36.0 & 3.9 & 44.4 & 15.4 & 0.2 & $0.2^{\mathrm{b}}$ \\
\hline 2 positive (A2) & 14 & 21.4 & 14.3 & 28.6 & 71.4 & 0 & 0 \\
\hline 3 positive (A3) & 18 & 0 & 0 & 27.8 & $55.6^{\mathrm{a}}$ & 5.6 & 11.1 \\
\hline Always positive (A4) & 39 & 2.6 & 0 & 20.5 & 25.6 & $17.9^{\mathrm{a}}$ & $33.3^{\mathrm{a}}$ \\
\hline
\end{tabular}

${ }^{\mathrm{a}} P<0.05$, serological pattern positively associated with fecal shedding pattern.

${ }^{\mathrm{b}} P<0.05$, serological pattern negatively associated with fecal shedding pattern.

${ }^{1} \mathrm{CS}=$ constant shedder; FS = fluctuating shedder; NCS = never confidently shedder; NON-P = non-progressor; PRO = progressor; UN = unclassifiable.

\section{DISCUSSION}

This study provides unique data on MAP fecal shedding and its association with serological patterns. First, we present a more accurate description of fecal shedding patterns by using qPCR, which is a more sensitive method for detecting MAP than culture because it enables the detection of non-culturable amounts of MAP shed in feces. Thus, the proportion of animals of the final study sample found to be shedders is likely higher than what would be found by FC. For example, the intermediate persistent shedder category reported here fluctuated between shedding and low or nonculturable shedding assessed by qPCR, but was never negative. This pattern would likely have gone unnoticed by FC. The fact that we monitored a large number of animals from many different herds 4 times over approximately 1 yr provided us with extra detail on the MAP fecal shedding patterns in natural infections. The present study included a large number of herds (21 herds completed the follow-up), in contrast to other studies that surveyed one or a few herds (Benedictus et al., 2008; Nielsen, 2008; Schukken et al., 2015). Using a large number of herds allowed us to show the diversity of scenarios in high seroprevalence herds, and demonstrate that even in these contaminated environments, some animals remained non-shedders over a year ( $11 \%$ of the final study sample, but $>30 \%$ in some herds). However, in other herds all animals were fecal shedders at least once during the year.

Although $1 \mathrm{yr}$ is a limited time frame compared with other longitudinal studies that encompassed several years or even decades (such as Benedictus et al., 2008), it was our objective to focus on a realistic time period to aid in the farmers' decision-making and the control of disease. Despite the high value of longitudinal field data highlighted by previous studies (Mitchell et al., 2015b; Schukken et al., 2015), longitudinal field studies of MAP in dairy cattle continue to be scarce. The originality of this labor- and resource-intensive study resides in the sampling frequency of both feces and sera in combination with the total duration, and the benefit of $\mathrm{qPCR}$ testing and the recognition of various $\mathrm{qPCR}$ categories that are differently interpreted.

Fecal culture is the closest to a gold standard for antemortem diagnosis, despite its low sensitivity [especially in animals that are intermittently shedding or shedding at low concentrations (Visser, 1999)]. It is noteworthy that sometimes variations in the semiquantitative FC method exist between laboratories (for example, culture on 4 tubes versus 3 tubes), which can also influence the outcome of this test (Crossley et al., 2005). Although FC is sometimes considered the gold standard, qPCR and ELISA are the surveillance tools used most often (Delafosse et al., 2019; Geraghty et al., 2014). Quantitative PCR is less laborious than FC and less time- and resource-consuming, thus providing numerous advantages. Quantitative PCR assays have shown a superior sensitivity (77.6; 95\% CI: 73.2-82.0) to culture on fecal samples from previously identified infectious cows (Laurin et al., 2015).

When using qPCR to detect MAP in feces, the passive shedding or "pass-through" phenomenon, and the ability of qPCR to detect DNA from nonviable bacteria must be considered. For this reason, some studies choose a cutoff $\mathrm{C}_{t}$ value that optimizes the accuracy of the qPCR by comparing results to those of a reference diagnostic test, as we did for the present study. Although this procedure is generally well founded, it may cancel out some of the advantages of $\mathrm{qPCR}$ by equalizing results with the less sensitive FC. The qPCR results from Kralik et al. (2014) showed that the amount of MAP shed by passive shedders is comparable to that shed by truly infected low shedders. In addition, because neither FC nor tissue culture is highly sensitive (Pavlik et al., 2000), a truly infected animal could be MAP-negative by culture (tissue or fecal) but positive by qPCR.

In our study, a considerable number of animals - almost $20 \%$ of the final study sample - were positioned at 
least once between the adjusted and the standard cutoff value without ever being confidently positive. Given the global interest in the control of paratuberculosis and the efforts put into this mission, this fraction of the cattle population cannot be neglected. A biologically sensible interpretation of results in this $\mathrm{C}_{t}$ range is needed; otherwise, potentially important information may be lost by assigning those animals to non-shedder status. If animals in this $\mathrm{C}_{t}$ range were passive shedders, we would expect their proportion to be smaller than that of shedders. This happened in 9 herds, but in 13 herds the proportion of "low or nonculturable shedders" surpassed the proportion of "confident shedders" $\left(\mathrm{C}_{t}<37\right)$. We cannot rule out the possibility that passive shedders are being included in the $\mathrm{C}_{t}$ range 37 to 40, but herds with a high proportion of animals in this $\mathrm{C}_{\mathrm{t}}$ range must be composed at least partially of true low shedders. The proportion of animals in the $\mathrm{C}_{\mathrm{t}}$ range 37 to 40 varied largely between herds (Table 1) but also within herds, in the different phases of the study (data not shown). This finding also suggests intermittent shedding due to a true infection rather than a pass-through. Another finding that supports the link between the $\mathrm{C}_{\mathrm{t}}$ range 37 to 40 and "real" shedding is that herd I was the only herd with no never confidently shedders (animals with $\mathrm{C}_{\mathrm{t}}>37$ at all times) and also the herd with the largest proportion of animals that shed at least twice consecutively $(+$ or $*)$.

Missing the diagnosis of a low shedder implies keeping an infectious animal on the farm that will likely stay undetected (subclinical shedding) until at least the next sampling event. The use of $\mathrm{qPCR}$ and the establishment of a category such as "low or nonculturable MAP shedder" may fill in this gap. Future research should explore whether this qPCR category can be an early predictor of a "confidently positive" qPCR result or a positive FC result, either alone or in combination with other tests and animal information. For farmers that implement test-and-cull strategies, it is advisable that labs report qPCR results with mention to the standard and the adjusted cutoff value, as well as the cutoff value indicative of high shedding. A semiquantitative interpretation of consecutive tests would provide useful information for decisions and for prioritizing the culling of certain animals.

In the present study, the semiquantitative use of $\mathrm{qPCR}$ was crucial for establishing a category of high shedding and describing progression patterns. The cutoff value we obtained to refer to high shedders $(<27.23)$ was in the range of results obtained by other studies. For example, Leite et al. (2013) reported that high FC counts ( $>100 \mathrm{cfu} /$ tube, same criterion we used) was mainly related to $\mathrm{C}_{\mathrm{t}}$ values between 24 and 30 with the use of a qPCR targeting the IS900 gene. For the present study, we investigated a subset of 34 fecal samples that were too numerous to count by FC to determine the bacterial load by means of bacterial enumeration (data not shown). Out of these, 29 showed a value of $>1,000$ $\mathrm{cfu} / \mathrm{g}$, and thus were either heavy or super-shedders as defined by Fecteau and Whitlock (2010); our qPCR cutoff value for high shedding successfully detected 23 of those 29. Thus, the semiquantitative use of qPCR can aid in the detection of high shedders in control programs. Heavy shedders are thought to contribute largely to contamination of the farm environment, and they can also transmit MAP to other adults (Pradhan et al., 2011; Schukken et al., 2015). However, the magnitude of the effect of MAP super-shedders has been questioned (Mitchell et al., 2008; Slater et al., 2016), and other sources of infection likely affect the persistence of MAP in dairy herds. Given that young animals become infected with a lower infectious dose than adults (McKenna et al., 2006), that animals exposed to lower doses may require several years before the signs of infection develop (Begg and Whittington, 2008), and that this bacterium has high environmental persistence, undetected low shedders can be an explanation for the on-farm infection persistence and the inability to successfully eliminate the infection.

Although we grouped the 80 different qPCR profiles we obtained (that is, combination of results of 4 events) into 6 fecal shedding patterns, many other possibilities could have described fecal shedding that would yield more numerous patterns. For example, we considered "+" and "*" similarly, but one could take into account this variation to define further patterns that indicate reversal of positivity, transition from low or nonculturable shedding to confidently positive, and vice versa. However, this large heterogeneity would have resulted in a small number of animals showing the same pattern, and forced us to group their profiles using broad criteria. Some of the categories of fecal shedding we used were comparable to those used by Nielsen (2008): for example, never shedders. Nielsen (2008) reported that $79 \%$ of the cows were never MAP-positive by FC, whereas in our study only $11.6 \%$ of the cows were never shedders by qPCR. However, comparisons need to be made cautiously, because we targeted animals that were highly likely to be infected in herds with a high seroprevalence in the previous year and used a more sensitive detection technique.

Surprisingly, in our study we found a large diversity in the herd distribution of fecal shedding patterns (Figure 2). These results were encouraging, because in spite of showing a high seroprevalence in the previous year, some herds managed to have a high percentage of never shedders. The fact that the proportion of intermediate persistent shedders was higher in $1 \mathrm{yr}$ olds than in older 
animals suggests that this pattern was a transition from latency to fecal shedding, and that other fecal shedding patterns would be established in those animals later in life.

With respect to continuity of fecal shedding, Mitchell et al. (2015a) reported that in natural infections $<5 \%$ of cows switched between no shedding and low shedding as assessed by FC (defined as "intermittent shedders," but in this paper referred to as "non-continuous shedders"). Conversely, in the cows we surveyed, we detected a high rate of animals that switched to qPCR negativity after being positive. In addition, almost $23 \%$ of the animals showed all 3 statuses assessable by qPCR $\left(+,{ }^{*},-\right)$ over the study period. Our selection of high-prevalence herds was likely to be responsible for the higher observed infection proportion, as well as the use of $\mathrm{qPCR}$ rather than $\mathrm{FC}$, because $\mathrm{qPCR}$ is better at detecting switches from negativity to low shedding. The increased percentage of animals at age $8 \mathrm{yr}$ that switched from negativity to positivity by the last sampling event (---+ and $--{ }^{*}$ profiles) suggests the existence of a tipping point, at which animals that were experiencing a long incubation period start shedding MAP in feces. This has to be taken into account when considering whether to keep a cow older than 8 to $9 \mathrm{yr}$.

As expected because of the heterogeneity of MAP infection states, only a small percentage of the studied animals were progressors. However, we were surprised to find that animals aged $1 \mathrm{yr}$ at the start of the study developed high shedding and even transitioned to progression. According to Mitchell et al. (2015a), naturally infected cows typically become high shedders after age 5 yr. The finding that cows that became primiparous during the study were more frequently non-progressors suggests the existence of 2 distinct cow populations: animals whose pattern is established before the first calving and animals that transition to their progression pattern afterward. These results show the importance of surveying young and nulliparous cows in highprevalence herds, and the utility of a semiquantitative interpretation of qPCR to target the removal of young animals that are high shedders.

For all these fecal shedding traits, we found that patterns that implied a switch in qPCR category were most frequent. Given that a single positive qPCR result most likely reflects a transitory status, decision-making to find a compromise between disease control and economic factors is further complicated. Continuity of fecal shedding may be a better indicator of a trend toward persistence of infection, because animals that shed $\left(\mathrm{C}_{\mathrm{t}}\right.$ $<40$ ) at least twice consecutively rarely reverse to a durable negativity. High shedders also rarely revert to negativity (only 1 case). Thus, an approach might be to test for fecal shedding by qPCR, cull animals that are high shedders, and then perform a second fecal qPCR of positive animals within 4 mo to cull those that are positive a second time. Conversely, a qPCR result with $\mathrm{C}_{\mathrm{t}}>37$ is highly indicative of the absence of high shedding in the following months.

The fact that more than $80 \%$ of the final study sample was ELISA-negative 4 times, whereas $68 \%$ were qPCR confidently positive at least once, is clearly indicative of the mismatch between serology and qPCR. This finding was in line with the results of Schukken et al. (2015), in which only $1.4 \%$ of samples were serologically positive, whereas $16.7 \%$ of tissue samples at slaughter were culture-positive. In fact, to overcome this mismatch between ELISA and qPCR, adjustment of the ELISA cutoff value may be necessary, as implemented by Jubb (2005) and Delafosse et al. (2019), among others. Also the establishment of multiple cutoff values (and thus of categories other than "positive" and "negative") could be helpful in finding a link between serology results and fecal shedding. Because ELISA is a tool commonly used in control programs and is affordable, finding a correlation between serological and fecal shedding patterns is of major interest. Although imperfect, ELISA can be used to detect major patterns. We found that quarterly serological tests over a year can be used to detect animals that are shedding continuously, in high quantities, or both. This is important, because those shedding patterns rarely reverse to negativity. The serological pattern A4 in particular indicated persistence of fecal shedding and high shedding. In line with this, Schukken et al. (2015) found an increase in optical density values in progressors as the counts increased. Serological pattern A3 was also related to persistent and continuous shedding, but not to high shedding. Thus, by the third ELISA-positive sample, one can strongly recommend removing the animal. Because it is not practical or realistic for a commercial farm to collect and test 3 blood samples per year, ELISA tests could be implemented on individual milk samples. These are easier to collect and cheaper to test. Of course, implementation of such a sampling schedule depends on the economic value of the individual and the presence of a coherent test-andcull strategy in a farm. In the case of farms that are highly infected and have just started a control plan, we advise prioritizing the removal of progressors by detecting them with a fourth positive ELISA test. A pattern of 4 times negative (A0) conversely indicates a low probability of persistent shedding and of progression.

Individual milk ELISA patterns have been described by Nielsen (2008) to explain the probability of a cow testing positive by $\mathrm{FC}$ after a positive ELISA result. That study found that 2 or more ELISA-positive tests 
suggested a higher probability of fecal shedding, similar to our findings in serological patterns. Beaver et al. (2017) found a strong correlation between individual milk ELISA positivity and positive fecal $\mathrm{qPCR}$ in progressors from 2 low-prevalence herds. Further research is needed in different scenarios (for example, high-prevalence herds) to elucidate the utility of individual milk ELISA to identify fecal shedding patterns. Individual milk ELISA can be an interesting alternative to serum ELISA, but Eisenberg et al. (2015) report that changes in milk yield strongly influence the concentration of MAP antibodies in milk, among other limitations.

For the present study, we enrolled commercial dairy farms that functioned normally. The research team did not interfere in any of the farmers' decisions, and the farmers did not receive the test results until the end of the study. Thus, we do not believe that positive animals, especially high shedders, were underrepresented because of early culling. Infected animals could have been more likely to be removed because of decreased milk production and decreased fertility, but this would also have been the case in commercial herds that did not have a test-and-cull regimen in place. However, we cannot know how differences in disease management and decisions may have affected the composition of our study sample and the outcomes of the study. Some effects may be masked by differences in herd conditions, particularly the prevalence and percentage of high shedders, as well as herd size, because larger herds were more likely to contribute more animals to the final study sample. This may also explain the large variation in pattern distribution between herds, even though all herds had high seroprevalence the year before the study.

This study provides baseline data for the identification of early predictors of fecal shedding patterns and high shedding. The ability to detect highly infectious animals early and predict their future fecal shedding in a short time frame would help in the management of disease in the farm population and enable rapid responses to prevent the spread and persistence of the pathogen. In particular, we envision complementing these data with further research into which combination of herd-level and cow-level variables (diagnostic tests and individual characteristics) can best predict high and persistent shedding to aid in farmers' decision-making and advise in the control programs. In contrast to experimental studies that provided detailed data on multiple immunological assays that were not currently in place in France, we obtained relevant data with diagnostic tools already implemented by French regional control programs (Coursaget, 2009); thus, the results from our study have a high potential for direct applicability.

\section{ACKNOWLEDGMENTS}

The data from the national database SNIG were contributed by the French departmental agriculture agencies, the performance-recording organizations institutions, and the artificial insemination centers. The authors acknowledge funding from the INRA metaprogram "Integrated Management of Animal Health" for the "GISA-PICSAR" project, from the Regional Council of Pays de la Loire, from APIS-GENE, and GDS France for the "PARADIGM" Project. The funders had no role in the production, analysis, interpretation, or reporting of the data. The authors also thank 2 anonymous reviewers for their useful contributions.

\section{REFERENCES}

Beaver, A., R. W. Sweeney, E. Hovingh, D. R. Wolfgang, Y. T. Gröhn, and Y. H. Schukken. 2017. Longitudinal relationship between fecal culture, fecal quantitative PCR, and milk ELISA in Mycobacterium avium ssp. paratuberculosis-infected cows from low-prevalence dairy herds. J. Dairy Sci. 100:7507-7521. https://doi.org/10.3168/ jds.2017-12928.

Begg, D. J., and R. J. Whittington. 2008. Experimental animal infections models for Johne's disease, an infectious enteropathy caused by Mycobacterium avium subsp. paratuberculosis. Vet. J. 176:129145. https://doi.org/10.1016/j.tvjl.2007.02.022.

Benedictus, A., R. M. Mitchell, M. Linde-Widmann, R. Sweeney, T. Fyock, Y. H. Schukken, and R. H. Whitlock. 2008. Transmission parameters of Mycobacterium avium subspecies paratuberculosis infections in a dairy herd going through a control program. Prev. Vet. Med. 83:215-227. https://doi.org/10.1016/j.prevetmed.2007 .07 .008 .

Collins, M. T. 2003. Paratuberculosis: Review of present knowledge Acta Vet. Scand. 44:217-221.

Coursaget, S. 2009. Etat des lieux de l'offre des actions de maitrise de la paratuberculose bovine dans le Grand Ouest. MS Thesis. Nantes Atlantic National College of Veterinary Medicine, Food Science and Engineering, France. [In French].

Crossley, B. M., F. J. Zagmutt-Vergara, T. L. Fyock, R. H. Whitlock, and I. A. Gardner. 2005. Fecal shedding of Mycobacterium avium ssp. paratuberculosis by dairy cows. Vet. Microbiol. 107:257-263. https://doi.org/10.1016/j.vetmic.2005.01.017.

Delafosse, A., E. Meens, T. Rambaud, F. Hanoy, and H. Achour. 2019. Sensitivities of a bulk-tank milk ELISA and composite fecal qPCR to detect various seroprevalence levels of paratuberculosis in cattle herds in Normandy, France. Can. Vet. J. 60:275-281.

Eisenberg, S. W. F., E. Veldman, V. P. M. G. Rutten, and A. P. Koets. 2015. A longitudinal study of factors influencing the result of a Mycobacterium avium ssp. paratuberculosis antibody ELISA in milk of dairy cows. J. Dairy Sci. 98:2345-2355. https://doi.org/10 $.3168 /$ jds.2014-8380.

Fecteau, M.-E., and R. H. Whitlock. 2010. Paratuberculosis in cattle. Pages 144-146 in Paratuberculosis: organism, disease, control. M. A. Behr and D. M. Collins, ed. CABI, Wallingford, UK.

Geraghty, T., D. A. Graham, P. Mullowney, and S. J. More. 2014. A review of bovine Johne's disease control activities in 6 endemically infected countries. Prev. Vet. Med. 116:1-11. https://doi.org/10 .1016/j.prevetmed.2014.06.003.

Hofmann, H. 2008. Mosaic plots and their variants. Pages $617-642$ in Handbook of Data Visualization. C. Chen, W. Härdle, and A. Unwin, ed. Springer, Berlin, Germany.

Jeppson, H., H. Hofmann, and D. Cook. 2017. ggmosaic: Mosaic Plots in the "ggplot2" Framework. R package version 0.1.2. Accessed Jul. 7, 2019. https://CRAN.R-project.org/package=ggmosaic 
Jubb, T. F. 2005. The effect of interpreting an ELISA at a lower cut-off on detection of clinical cases of bovine Johne' s disease. Aust. Vet. J. 83:305-307. https://doi.org/10.1111/j.1751-0813 .2005.tb12749.x.

Khol, J. L., E. Geisbauer, M. Wassertheurer, S. Revilla-Fernández, J. Damoser, E. Österreicher, M. Dünser, U. Kleb, and W. Baumgartner. 2012. Outcome of three commercial serum ELISAs and faecal detection of Mycobacterium avium ssp. paratuberculosis in consecutive samples from a cattle herd with low prevalence of paratuberculosis (Johne's disease). Transbound. Emerg. Dis. 59:197-207. https://doi.org/10.1111/j.1865-1682.2011.01256.x.

Kralik, P., R. Pribylova-Dziedzinska, A. Kralova, K. Kovarcik, and I. Slana. 2014. Evidence of passive faecal shedding of Mycobacterium avium ssp. paratuberculosis in a Limousin cattle herd. Vet. J. 201:91-94. https://doi.org/10.1016/j.tvjl.2014.02.011.

Kralik, P., and M. Ricchi. 2017. A basic guide to real time PCR in microbial diagnostics: Definitions, parameters, and everything. Front. Microbiol. 8:108. https://doi.org/10.3389/fmicb.2017.00108.

Laurin, E. L., M. Chaffer, J. T. McClure, S. L. B. McKenna, and G. P. Keefe. 2015. The association of detection method, season, and lactation stage on identification of fecal shedding in Mycobacterium avium ssp. paratuberculosis infectious dairy cows. J. Dairy Sci. 98:211-220. https://doi.org/10.3168/jds.2014-8406.

Leite, F. L., K. D. Stokes, S. Robbe-Austerman, and J. R. Stabel. 2013. Comparison of fecal DNA extraction kits for the detection of Mycobacterium avium ssp. paratuberculosis by polymerase chain reaction. J. Vet. Diagn. Invest. 25:27-34. https://doi.org/10.1177/ 1040638712466395.

Mandrekar, J. N. 2010. Receiver operating characteristic curve in diagnostic test assessment. J. Thorac. Oncol. 5:1315-1316. https:// doi.org/10.1097/JTO.0b013e3181ec173d.

McKenna, S. L. B., G. P. Keefe, A. Tiwari, J. Vanleeuwen, and H. W. Barkema. 2006. Johne's disease in Canada part II: Disease impacts, risk factors and control programs for dairy producers. Can. Vet. J. 47:1089-1099.

Meyer, D., A. Zeileis, and K. Hornik. 2016. vcd: Visualizing Categorical Data. R package version 1.4-3. Accessed Jul. 7, 2019. https:// cran.r-project.org/web/packages/vcd/vcd.pdf.

Mitchell, R. M., Y. Schukken, A. Koets, M. Weber, D. Bakker, J. Stabel, R. H. Whitlock, and Y. Louzoun. 2015a. Differences in intermittent and continuous fecal shedding patterns between natural and experimental Mycobacterium avium subspecies paratuberculosis infections in cattle. Vet. Res. 46:66. https://doi.org/10.1186/ s13567-015-0188-x.

Mitchell, R. M., R. H. Whitlock, Y. T. Gröhn, and Y. H. Schukken. 2015b. Back to the real world: Connecting models with data. Prev. Vet. Med. 118:215-225. https://doi.org/10.1016/j.prevetmed.2014 .12 .009 .

Mitchell, R. M., R. H. Whitlock, S. M. Stehman, A. Benedictus, P. P. Chapagain, Y. T. Grohn, and Y. H. Schukken. 2008. Simulation modeling to evaluate the persistence of Mycobacterium avium ssp. paratuberculosis (MAP) on commercial dairy farms in the United States. Prev. Vet. Med. 83:360-380. https://doi.org/10.1016/j prevetmed.2007.09.006.

Nielsen, S. S. 2008. Transitions in diagnostic tests used for detection of Mycobacterium avium ssp. paratuberculosis infections in cattle. Vet. Microbiol. 132:274-282. https://doi.org/10.1016/j.vetmic .2008.05.018.

Nielsen, S. S., and N. Toft. 2008. Ante mortem diagnosis of paratuberculosis: A review of accuracies of ELISA, interferon-gamma assay and faecal culture techniques. Vet. Microbiol. 129:217-235. https:/ /doi.org/10.1016/j.vetmic.2007.12.011.

Pavlik, I., L. Matlova, J. Bartl, P. Svastova, L. Dvorska, and R. Whitlock. 2000. Parallel faecal and organ Mycobacterium avium ssp. paratuberculosis culture of different productivity types of cattle. Vet. Microbiol. 77:309-324. https://doi.org/10.1016/S0378 $-1135(00) 00316-3$.

Pradhan, A. K., R. M. Mitchell, A. J. Kramer, M. J. Zurakowski, T. L. Fyock, R. H. Whitlock, J. M. Smith, E. Hovingh, J. A. S. Van Kessel, J. S. Karns, and Y. H. Schukken. 2011. Molecular epidemiology of Mycobacterium avium ssp. paratuberculosis in a longitudinal study of three dairy herds. J. Clin. Microbiol. 49:893-901. https:/ /doi.org/10.1128/JCM.01107-10.

R Core Team. 2016. R: A language and environment for statistical computing. R Foundation for Statistical Computing, Vienna, Austria. Accessed Jul. 7, 2019. https://www.R-project.org/.

Robin, X., N. Turck, A. Hainard, N. Tiberti, F. Lisacek, J.-C. Sanchez, and M. Müller. 2011. pROC: An open-source package for R and $\mathrm{S}+$ to analyze and compare ROC curves. BMC Bioinformatics 12:77. https://doi.org/10.1186/1471-2105-12-77.

Schukken, Y. H., R. H. Whitlock, D. Wolfgang, Y. Grohn, A. Beaver, J. VanKessel, M. Zurakowski, and R. Mitchell. 2015. Longitudinal data collection of Mycobacterium avium subspecies paratuberculosis infections in dairy herds: The value of precise field data. Vet. Res. 46:65. https://doi.org/10.1186/s13567-015-0187-y.

Slater, N., R. M. Mitchell, R. H. Whitlock, T. Fyock, A. K. Pradhan, E. Knupfer, Y. H. Schukken, and Y. Louzoun. 2016. Impact of the shedding level on transmission of persistent infections in Mycobacterium avium subspecies paratuberculosis (MAP). Vet. Res. 47:38. https://doi.org/10.1186/s13567-016-0323-3.

Sweeney, R. W. 2011. Pathogenesis of paratuberculosis. Vet. Clin. North Am. Food Anim. Pract. 27:537-546. https://doi.org/10 .1016/j.cvfa.2011.07.001.

Therneau, T., B. Atkinson, and B. Ripley. 2015. rpart: Recursive Partitioning and Regression Trees. $\mathrm{R}$ package version 4.1-10. Accessed Jul. 7, 2019. https://CRAN.R-project.org/package=rpart

Visser, I. 1999. Reproducibility of a faecal culture method for Mycobacterium avium subsp. paratuberculosis. Page 51 in Proceedings of the 6th International Colloquium on Paratuberculosis. Melbourne, Australia.

Waddell, L. A., A. Rajic, K. D. C. Stärk, and S. A. Mcewen. 2016. The potential public health impact of Mycobacterium avium ssp. paratuberculosis: Global opinion survey of topic specialists. Zoonoses Public Health 63:212-222. https://doi.org/10.1111/zph.12221.

Wang, C., B. W. Turnbull, Y. T. Grohn, and S. S. Nielsen. 2006. Estimating receiver operating characteristics curves with covariates when there is no perfect reference test for diagnosis of Johne's disease. J. Dairy Sci. 89:3038-3046. https://doi.org/10.3168/jds .S0022-0302(06)72577-2.

Whipple, D. L., D. R. Callihan, and J. L. Jarnagin. 1991. Cultivation of Mycobacterium paratuberculosis from bovine fecal specimens and a suggested standardized procedure. J. Vet. Diagn. Invest. 3:368-373. https://doi.org/10.1177/104063879100300424.

Whitlock, R. H., S. J. Wells, R. W. Sweeney, and J. Van Tiem. 2000. ELISA and fecal culture for paratuberculosis (Johne's disease): Sensitivity and specificity of each method. Vet. Microbiol. 77:387398. https://doi.org/10.1016/S0378-1135(00)00324-2.

Wickham, H. 2009. Ggplot2: Elegant Graphics for Data Analysis. Springer-Verlag, New York.

Zeileis, A., D. Meyer, and K. Hornik. 2007. Residual-based shadings for visualizing (conditional) independence. J. Comput. Graph. Stat. 16:507-525. https://doi.org/10.1198/106186007X237856.

Zeileis, A., D. Meyer, and K. Hornik. 2017. Residual-based shadings in vcd. Accessed Jul. 7, 2019. https://cran.r-project.org/web/ packages/vcd/vignettes/residual-shadings.pdf. 